\title{
The Problem of Invariance for Covariant Hamiltonians (*).
}

\author{
J. Muñoz Masqué (**) - M. Eugenia Rosado María (***)
}

ABSTRACT - The problem of invariance for the covariant Hamiltonians attached to first-order Lagrangian densities on the bundle of connections of a principal bundle and to first- and second-order Lagrangian densities on linear frame bundles, is solved.

\section{Position of the problem.}

In classical Mechanics, the Hamiltonian function attached to the Lagrangian density $\Lambda=L\left(t, q^{i}, \dot{q}^{i}\right) d t$ on $J^{1}(\mathbb{R}, Q) \simeq \mathbb{R} \times T Q$ is given by,

$$
H=\dot{q}^{i} \frac{\partial L}{\partial \dot{q}^{i}}-L .
$$

If $\Theta_{\Lambda}$ denotes the Poincaré-Cartan form attached to $\Lambda$, then

$$
\begin{aligned}
\Theta_{\Lambda} & =p_{i} d q^{i}-H d t \\
& =\sigma(L)^{*} \kappa-H d t
\end{aligned}
$$

where $\sigma(L): \mathbb{R} \times T Q \rightarrow \mathbb{R} \times T^{*} Q$ is the Legendre transformation, $p_{i}=\partial L / \partial \dot{q}^{i}$, and $\kappa$ is the canonical 1-form on $T^{*} Q$.

As it was early observed in [9], the Hamiltonian function is not an invariant concept when an arbitrary fibred manifold $t: E \rightarrow \mathbb{R}$ is considered, generalizing the notion of «absolute time» (e.g., see $[4,15,13]$ for a general

(*) Supported by Ministerio of Ciencia y Tecnología of Spain, under grant \#MTM2005-00173.

(**) Indirizzo dell'A.: Instituto de Física Aplicada, CSIC C/ Serrano 144, 28006-Madrid, Spain

E-mail: jaime@iec.csic.es

(***) Department of Applied Mathematics «G. Sansone», University of Florence, Via S. Marta 3, I-50139 Florence, Italy

E-mail: rosado@dma.unifi.it 
development from this point of view), instead of the trivial direct product bundle $\mathbb{R} \times Q \rightarrow \mathbb{R}$. In this case, an Ehresmann connection is needed in order to lift the vector field $\partial / \partial t$ from $\mathbb{R}$ to $E$. Then, the Hamiltonian function is defined by contracting $\Theta_{\Lambda}$ with the horizontal lift of $\partial / \partial t$.

In the field theory - where no distinguished vector field exists on the ground manifold - the need of an Ehresmann connection is even greater, in order to attach a covariant Hamiltonian to each Lagrangian density; for example, see [13, 4.1], [14], and the definitions below.

Let $p: M \rightarrow N$ be a fibred manifold over a connected manifold $N$, with $n=\operatorname{dim} N, \operatorname{dim} M=m+n$, oriented by a volume form $\boldsymbol{v}$. Throughout the paper, Latin (resp. Greek) indices run from 1 to $n$ (resp. $m$ ).

We consider only coordinate systems $\left(x^{i}\right)$ on $N$ adapted to $\boldsymbol{v}$, that is

$$
\boldsymbol{v}=d x^{1} \wedge \cdots \wedge d x^{n}, \quad \boldsymbol{v}_{i}=d x^{1} \wedge \cdots \wedge \widehat{d x^{i}} \wedge \cdots \wedge d x^{n} .
$$

Let $\gamma$ be an Ehresmann (or non-linear) connection on $p$, i.e., $\gamma$ is a differential 1-form on $M$ with values in the vertical bundle $V(p)$, such that $\gamma(X)=X, \forall X \in V(p)$ (cf. [13, 2.2]). According to [14], the covariant Hamiltonian $\Lambda^{\gamma}$ associated to a Lagrangian density on $J^{1} M, \Lambda=L \boldsymbol{v}$, $L \in C^{\infty}\left(J^{1} M\right)$, with respect to $\gamma$ is the Lagrangian density defined by

$$
\Lambda^{\gamma}=\left(\left(p^{10}\right)^{*} \gamma-\vartheta^{1}\right) \wedge \omega_{\Lambda}-\Lambda,
$$

where, $p^{10}: J^{1} M \rightarrow M$ denotes the natural projection, $\vartheta^{1}$ is the $V(p)$-valued 1 -form on $J^{1} M$ governing the contact structure on the jet bundle, which is given on a fibred coordinate system $\left(x^{i}, y^{\alpha}\right)$, by $\vartheta^{1}=\left(d y^{\alpha}-y_{i}^{\alpha} d x^{i}\right) \otimes \partial / \partial y^{\alpha}$ (also see Section 3 below), and $\omega_{A}$ is the Legendre form attached to $\Lambda$, i.e., the $V^{*}(p)$-valued $p^{1}$-horizontal $(n-1)$-form on $J^{1} M$ given by

$$
\omega_{\Lambda}=(-1)^{i-1} \frac{\partial L}{\partial y_{i}^{\alpha}} \boldsymbol{v}_{i} \otimes d y^{\alpha},
$$

where $\left(x^{i}, y^{\alpha} ; y_{i}^{\alpha}\right)$ is the coordinate system induced from $\left(x^{i}, y^{\alpha}\right)$ on the 1 -jet bundle (see Section 2.1 below for the details). Locally, we have

$$
\Lambda^{\gamma}=\left(\left(\gamma_{i}^{\alpha}+y_{i}^{\alpha}\right) \frac{\partial L}{\partial y_{i}^{\alpha}}-L\right) \boldsymbol{v} .
$$

From (3) we obtain the decomposition of the Poincaré-Cartan form analogous to (1), i.e.,

$$
\begin{aligned}
\Theta_{\Lambda} & =\vartheta^{1} \wedge \omega_{\Lambda}+\Lambda \\
& =\left(p^{10}\right)^{*} \gamma \wedge \omega_{\Lambda}-\Lambda^{\gamma} .
\end{aligned}
$$


An automorphism of the fibred manifold $p: M \rightarrow N$ is a diffeomorphism $\Phi: M \rightarrow M$ for which a diffeomorphism exists on the ground manifold $\phi: N \rightarrow N$ such that $p \circ \Phi=\phi \circ p$. The set of such automorphisms is denoted by $\operatorname{Aut}(p)$ and its "Lie algebra" is the space $\operatorname{Aut}(p) \subset \mathfrak{X}(M)$ of $p$ projectable vector fields on $M$, or equivalently, a vector field belongs to $\operatorname{Aut}(p)$ if and only if each transformation $\Phi_{t}$ of its flow, belongs to Aut $(p)$.

Given a subgroup $\mathcal{G} \subseteq \operatorname{Aut}(p)$, a Lagrangian density $\Lambda$ is said to be $\mathcal{G}$ invariant if

$$
\left(\Phi^{(1)}\right)^{*} \Lambda=\Lambda, \quad \forall \Phi \in \mathcal{G},
$$

where $\Phi^{(1)}: J^{1} M \rightarrow J^{1} M$ denotes the 1 -jet prolongation of $\Phi$. Infinitesimally, the equation (6) can be reformulated as follows:

$$
L_{X^{(1)}} \Lambda=0, \quad \forall X \in \operatorname{Lie}(\mathcal{G}) .
$$

When a group $\mathcal{G}$ of transformations of $M$ is given, a natural question arises:

(Q) Does a class of connections $\gamma$ exist such that $\Lambda^{\gamma}$ is $\mathcal{G}$-invariant for every $\mathcal{G}$-invariant Lagrangian density $\Lambda$ ?

A natural answer to this question is to assume that the connection $\gamma$, itself, is $\mathcal{G}$-invariant. In fact, for every $X \in \operatorname{Aut}(p)$ we can define the Lie derivative of $\gamma$ with respect to $X$ as follows (see $[6, \S \mathrm{XI}]): L_{X} \gamma(Y)=[X, \gamma(Y)]-\gamma([X, Y])$, for every $Y \in \mathfrak{X}(M)$, and the infinitesimal $\mathcal{G}$-invariance of $\gamma$ means $L_{X} \gamma=0$, $\forall X \in \operatorname{Lie}(\mathcal{G})$. Then, the following result holds:

Proposition 1.1. If $\gamma$ is infinitesimally $\mathcal{G}$-invariant, then $\gamma$ solves the question (Q), i.e., $L_{X^{(1)}} \Lambda=0, \forall X \in \operatorname{Lie}(\mathcal{G})$, implies $L_{X^{(1)}} \Lambda^{\gamma}=0$, $\forall X \in \operatorname{Lie}(\mathcal{G})$.

Proof. If $X=u^{i} \partial / \partial x^{i}+v^{\alpha} \partial / \partial y^{\alpha}, u^{i} \in C^{\infty}(N), v^{\alpha} \in C^{\infty}(M)$, is the local expression of $X \in \operatorname{Lie}(\mathcal{G})$, then from the general formulas (11), (12) in Section 2.2 below, we obtain in particular,

$$
X^{(1)}=u^{i} \frac{\partial}{\partial x^{i}}+v^{\alpha} \frac{\partial}{\partial y^{\alpha}}+v_{i}^{\alpha} \frac{\partial}{\partial y_{i}^{\alpha}}, \quad v_{i}^{\alpha}=\frac{\partial v^{\alpha}}{\partial x^{i}}+y_{i}^{\beta} \frac{\partial v^{\alpha}}{\partial y^{\beta}}-y_{j}^{\alpha} \frac{\partial w^{j}}{\partial x^{i}} .
$$

Furthermore, if $\gamma=\left(d y^{\alpha}+\gamma_{i}^{\alpha} d x^{i}\right) \otimes \partial / \partial y^{\alpha}, \gamma_{i}^{\alpha} \in C^{\infty}(M)$ is the local expression of the Ehresmann connection $\gamma$ (see the formula (13) in Section 3 below) we obtain $L_{X} \gamma=C_{i}^{\alpha}(X) d x^{i} \otimes \partial / \partial y^{\alpha}$, where

$$
C_{i}^{\alpha}(X)=X\left(\gamma_{i}^{\alpha}\right)+v_{i}^{\alpha}-\left(\gamma_{j}^{\beta}+y_{j}^{\beta}\right) \frac{\partial v_{i}^{\alpha}}{\partial y_{j}^{\beta}} .
$$


We remark on the fact that $L_{X} \gamma(Y)=0$ if $Y$ is a $p$-vertical vector field on $M$. Hence $L_{X} \gamma$ is $p$-horizontal, i.e., it defines a section of the vector bundle $p^{*} T^{*} N \otimes V(p)$.

Then, the formula (4) yields,

$$
\begin{aligned}
L_{X^{(1)}} \Lambda^{\gamma}= & \left\{\left(X\left(\gamma_{i}^{\alpha}\right)+v_{i}^{\alpha}\right) \frac{\partial L}{\partial y_{i}^{\alpha}}\right. \\
& \left.+\left(\gamma_{i}^{\alpha}+y_{i}^{\alpha}\right)\left(X^{(1)}\left(\frac{\partial L}{\partial y_{i}^{\alpha}}\right)+\frac{\partial L}{\partial y_{i}^{\alpha}} \frac{\partial u^{h}}{\partial x^{h}}\right)\right\} \boldsymbol{v} .
\end{aligned}
$$

As $\Lambda$ is $\mathcal{G}$-invariant, $X^{(1)} L=-L \partial u^{h} / \partial x^{h}$. Hence

$$
\left(\frac{\partial}{\partial y_{i}^{\alpha}} \circ X^{(1)}\right) L=-\frac{\partial L}{\partial y_{i}^{\alpha}} \frac{\partial u^{h}}{\partial x^{h}}
$$

Moreover, by computing the bracket $\left[\partial / \partial y_{i}^{\alpha}, X^{(1)}\right]$, from the previous formula we obtain

$$
X^{(1)}\left(\frac{\partial L}{\partial y_{i}^{\alpha}}\right)+\frac{\partial L}{\partial y_{i}^{\alpha}} \frac{\partial u^{h}}{\partial x^{h}}=\frac{\partial u^{i}}{\partial x^{h}} \frac{\partial L}{\partial y_{h}^{\alpha}}-\frac{\partial v^{\beta}}{\partial y^{\alpha}} \frac{\partial L}{\partial y_{i}^{\beta}},
$$

and substituting the right hand side of this equation for the left hand side into the equation (9) and taking the formula (8) into account, we finally obtain $L_{X^{(1)}} \Lambda^{\gamma}=C_{i}^{\alpha}(X)\left(\partial L / \partial y_{i}^{\alpha}\right) \boldsymbol{v}$

Unfortunately, the infinitesimal $\mathcal{G}$-invariance of $\gamma$ is a too restrictive condition to assure the existence of Ehresmann connections solving the question (Q). In fact, in general there is no solution to the system of equations $C_{i}^{\alpha}(X) \equiv 0, \forall X \in \operatorname{Lie}(\mathcal{G})$, where $C_{i}^{\alpha}(X)$ is given by the formula (8), as proved in the next proposition. First, some preliminaries are required.

Every $p$-projectable vector field $X \in \mathfrak{X}(M)$ vanishing at a point $y \in M$ induces a linear map $A_{X, y}: V_{y}(p) \rightarrow V_{y}(p)$ by setting $A_{X, y}(v)=[Y, X]_{y}$, $\forall v \in V_{y}(p)$, where $Y$ is any $p$-vertical vector field on $M$ such that $v=Y_{y}$.

Proposition 1.2. If for every $y \in M$, there exists a p-projectable vector field $X \in \operatorname{Lie}(\mathcal{G})$ such that $X_{y}=0, A_{X, y}=0, j_{x}^{1} X^{\prime}=0$, where $x=p(y)$ and $X^{\prime}$ is the projection of $X$ onto $N$, but $j_{y}^{1} X \neq 0$, then there is no infinitesimally $\mathcal{G}$-invariant Ehresmann connection on $p: M \rightarrow N$.

Proof. With the same notations as in the proof of Proposition 1.1, the assumption is equivalent to the following: $u^{i}(x)=0,\left(\partial u^{i} / \partial x^{j}\right)(x)=0$, $v^{\alpha}(y)=0,\left(\partial v^{\alpha} / \partial y^{\beta}\right)(y)=0$, for all indices $\alpha, \beta, i, j$, but $\left(\partial v^{\alpha} / \partial x^{i}\right)(y) \neq 0$ for 
some indices $\alpha, i$. Then, from the equation (8) for these indices, we obtain $C_{i}^{\alpha}(X)(y)=\left(\partial v^{\alpha} / \partial x^{i}\right)(y)$, thus leading us to a contradiction.

Fortunately, in the cases we shall deal with below, the vertical bundle $V(p)$ decomposes as $V(p) \cong p^{*} T^{*} N \otimes W$ for a certain vector bundle $W$, so that $L_{X} \gamma$ can be viewed as a section of the bundle $p^{*}\left(T^{*} N \otimes T^{*} N\right) \otimes W$, and the invariance of $\Lambda^{\gamma}$ is assured by the assumption of the vanishing of the antisymmetric part of the tensor field $L_{X} \gamma$ for every $X$. In these cases, there are plenty of Ehresmann connections answering the question (Q) above. Specifically, we deal with the following three cases: 1) First-order Lagrangian densities on the bundle of connections of a principal bundle; 2) First-order Lagrangian densities on the bundle of linear frames of a smooth manifold; and 3) The class of second-order Lagrangian densities on the bundle of linear frames of a smooth manifold, which admit a secondorder Hamiltonian formalism, i.e., second-order Lagrangian densities whose corresponding covariant Hamiltonian is also of second order. In the first and third cases, the question (Q) imposes an algebraic condition on the antisymmetric part of the horizontal part of the Ehresmann connection under consideration, but in the second case the solution to (Q) leads one to a true system of partial differential equations. This asymmetry should deserve a subsequent analysis.

Finally, a summary of the contents of the present work is as follows: in Section 2, the notations and preliminary results on jet bundles, contact transformations and Lagrangian densities, which are used below, are introduced. In Section 3, the notion af an Ehresmann connection of arbitrary order on a fibred manifold $p: M \rightarrow N$, is reviewed, several geometric properties of these non-linear connections are proved, and the action of the group Aut $(p)$ on the space of Ehresmann connections is defined. Section 4 is devoted to state the basics of diffeomorphism invariance on the bundle of linear frames $\pi: F N \rightarrow N$ of a smooth manifold $N$. Namely, we first reduce the problem of determining Diff $N$-invariant Lagrangian densities on $J^{r}(F N)$ to that of Diff $N$-invariant functions and then, we explain why Diff $N$-invariance can be reduced to consider only the infinitesimal notion of invariance; i.e., $\mathfrak{X}(N)$-invariance. Finally, bases (previously obtained) for $\mathfrak{X}(N)$-invariant functions for the cases $r=1,2$, are introduced. In Section 5 the invariance problem for first-order covariant Hamiltonians, is considered in two particular cases: First, for the bundle of connections on an arbitrary principal bundle under the gauge group of the bundle; second, for the bundles of linear frames under the group of diffeomorphism on the ground manifold. In both cases, a geo- 
metric characterization of Ehresmann connections providing invariant covariant Hamiltonians for every invariant Lagrangian, is given. In Section 6 a class of second-order Lagrangian densities admitting a second-order covariant Hamiltonian, is introduced. In Section 7, the invariance problem for second-order Lagrangian densities on linear frame bundles belonging to the class defined in Section 6, is solved for secondorder Ehresmann connections induced by a 2 -jet field, the explicit form of which is given in Theorem 7.4.

\section{Preliminaries and notations.}

\section{1 - Jet bundle notations.}

For every fibred manifold $p: M \rightarrow N$ there is a homomorphism $\operatorname{Aut}(p) \rightarrow \operatorname{Diff} N, \Phi \mapsto \phi$, whose kernel is the subgroup $\operatorname{Aut}^{v}(p)$ of fibred (or vertical) diffeomorphisms inducing the identity over $N$. A system of coordinates $\left(x^{i}, y^{\alpha}\right)$ on an open subset $U \subseteq M$ is said to be a fibred coordinate system for the submersion $p$ if $\left(x^{i}\right)$ is a coordinate system for $N$ on $p(U)$. The vertical bundle of $p$ is the sub-bundle $V(p)=\left\{X \in T M: p_{*} X=0\right\}$. Let $M_{x}=p^{-1}(x)$ be the fibre over $x$. We have $T_{y}\left(M_{x}\right)=V_{y}(M), \forall y \in M_{x}$; i.e., the vertical tangent vectors at $y$ are the vectors tangent to the fibre passing through $y$. If $\Phi: M \rightarrow M^{\prime}$ is a fibred map, then $\Phi_{*}: T M \rightarrow T M^{\prime}$ maps $V(p)$ into $V\left(p^{\prime}\right)$. Let $p^{k}: J^{k} M \rightarrow N$ be the $k$-jet bundle of local sections of $p: M \rightarrow N$, with natural projections $p^{k l}: J^{k} M \rightarrow J^{l} M, p^{k l}\left(j_{x}^{k} s\right)=j_{x}^{l} s$, for $k \geq l, j_{x}^{k} s$ denoting the $k$-jet at $x$ of a section $s$ of $p$ defined on a neighbourhood of $x \in N$. If $I=\left(i_{1}, \ldots, i_{n}\right) \in \mathbb{N}^{n}$ is a multi-index of order $|I|=i_{1}+\ldots+i_{n}$ then we set $\partial^{|I|} f / \partial x^{I}=\partial^{|I|} f /\left(\partial x^{1}\right)^{i_{1}} \ldots\left(\partial x^{n}\right)^{i_{n}}$, for every $f \in C^{\infty}\left(\mathbb{R}^{n}\right)$. Multi-indices are added and subtracted componentwise and $I \leq J$ means $i_{h} \leq j_{h}$ for $1 \leq h \leq n$. We set $I !=i_{1} ! \cdots i_{n}$ ! and $\left(\begin{array}{l}J \\ I\end{array}\right)=J ! /(J-I) ! I$ ! whenever $I \leq J$. We denote by $(i)$ the multi-index whose components are $(i)_{h}=\delta_{h}^{i}$, and we set $(i j)=(i)+(j), \quad(i j k)=$ $=(i)+(j)+(k)$, etc. Hence the symbol $\left(i_{1} \ldots i_{a}\right)$ thus defined depends symmetrically on the indices $i_{1}, \ldots, i_{a}$. The identity $\delta_{(j k)}^{(h i)}=\delta_{j}^{h} \delta_{k}^{i}+$ $+\delta_{k}^{h} \delta_{j}^{i}-\delta_{i}^{h} \delta_{k}^{j} \delta_{k}^{h}$ for Kronecker's deltas of multi-indices, will be used.

A fibred coordinate system $\left(x^{i}, y^{\alpha}\right)$ for $p$ on an open subset $U \subseteq M$, induces a coordinate system $\left(x^{i}, y_{I}^{\alpha}\right), 0 \leq|I| \leq r$, on $\left(p^{r 0}\right)^{-1}(U)=J^{r} U$; i.e., $y_{I}^{\alpha}\left(j_{x}^{r} s\right)=\left(\partial^{|I|}\left(y^{\alpha} \circ s\right) / \partial x^{I}\right)(x)$, with $y_{0}^{\alpha}=y^{\alpha}$. For first-order derivatives we simply write $y_{i}^{\alpha}$ instead of $y_{(i)}^{\alpha}$. Every fibred map $\Phi: M \rightarrow M^{\prime}$ whose induced 
map on the base manifolds $\phi: N \rightarrow N^{\prime}$ is a diffeomorphism, induces a map $\Phi^{(r)}: J^{r} M \rightarrow J^{r} M^{\prime}$ by setting $\Phi^{(r)}\left(j_{x}^{r} s\right)=j_{\phi(x)}^{r}\left(\Phi \circ s \circ \phi^{-1}\right)$.

Below, we make repeatedly use of the affine-bundle structure of the projection $p^{r, r-1}: J^{r} M \rightarrow J^{r-1} M$ modelled over the vector bundle

$$
\left(p^{r-1}\right)^{*}\left(p^{*} S^{r} T^{*} N \otimes V(p)\right)=S^{r} T^{*} N \otimes_{J^{r-1} M} V(p),
$$

which is expressed on a fibred coordinate system $\left(x^{i}, y^{\alpha}\right)$ as follows. We set $(d x)^{I}=\left(d x^{1}\right) \odot \stackrel{\left(i_{1}\right.}{\cdots} \odot\left(d x^{1}\right) \odot \ldots \odot\left(d x^{n}\right) \odot \stackrel{\left(i_{n}\right.}{\cdots} \odot\left(d x^{n}\right)$ for every multi-index $I=\left(i_{1}, \ldots, i_{n}\right)$, where the symbol $\odot$ stands for the symmetric product. If

$$
t_{r}=\sum_{|I|=r} \frac{1}{I !} \lambda_{I}(d x)_{x_{0}}^{I} \otimes\left(\partial / \partial y^{\alpha}\right)_{y_{0}}, x_{0}=p\left(y_{0}\right)
$$

is an element in $S^{r} T_{x_{0}}^{*} N \otimes V_{y_{0}}(p)$, then the $r$-jet $j_{x_{0}}^{r} s^{\prime}=t_{r}+j_{x_{0}}^{r} s$ is determined by the equations

$$
\begin{gathered}
j_{x_{0}}^{r-1} s^{\prime}=j_{x_{0}}^{r-1} s \\
\frac{\partial^{r}\left(y^{\alpha} \circ s^{\prime}\right)}{\partial x^{I}}\left(x_{0}\right)=\lambda_{I}+\frac{\partial^{r}\left(y^{\alpha} \circ s\right)}{\partial x^{I}}\left(x_{0}\right),|I|=r .
\end{gathered}
$$

2.2 - Contact forms and contact transformations.

Let $p: M \rightarrow N$ be a fibred manifold. A differential 1-form $\vartheta$ on $J^{r} M$ is said to be a contact form if $\left(j^{r} s\right)^{*} \vartheta=0$ for every local section $s$ of $p$. The set of contact forms is a sheaf of $C_{J^{r} M}^{\infty}$-modules, denoted by $\mathcal{C}_{M}^{r}$, which is locally spanned by the forms

$$
\vartheta_{I}^{\alpha}=d y_{I}^{\alpha}-y_{I+(i)}^{\alpha} d x^{i}, \quad|I| \leq r-1
$$

For every $X \in \mathfrak{X}(N), D_{X}: C^{\infty}\left(J^{r} M\right) \rightarrow C^{\infty}\left(J^{r+1} M\right)$ denotes the total derivative with respect to $X$, which is defined by $D_{X} f\left(j_{x}^{r+1} s\right)=X_{x}\left(f \circ j^{r} s\right)$ for every $f \in C^{\infty}\left(J^{r+1} M\right)$. The operator $D_{X}$ is the only derivation on $J^{\infty} M$ that satisfies: 1) $D_{X}$ projects onto $X$, and 2) $\vartheta_{I}^{\alpha}\left(D_{X}\right)=0$, for all $I, \alpha$. In particular, we denote the total derivative with respect to the coordinate $x^{i}$ by

$$
D_{i}=\frac{\partial}{\partial x^{i}}+\sum_{|I|=0}^{\infty} y_{I+(i)}^{\alpha} \frac{\partial}{\partial y_{I}^{\alpha}}
$$

We set $D^{I}=D_{1} \circ \stackrel{\left(i_{1}\right.}{\cdots} \circ D_{1} \circ \cdots \circ D_{n} \circ \stackrel{{ }_{i n}}{\cdots} \circ D_{n}$. Recall that $\left[D_{i}, D_{j}\right]=0$.

The horizontalization of a-possibly vector-valued-covariant tensor field 
$\tau$ of degree $q$ on $J^{r} M$ is the $p^{r+1}$-horizontal covariant tensor field hor $(\tau)$ of degree $q$ on $J^{r+1} M$ defined by hor $(\tau)\left(j_{x}^{r+1} s\right)=\left(p^{r+1}\right)^{*}\left(\left(j^{r} s\right)^{*} \tau\right)(x)$. The total differential of a $p^{r}$-horizontal form $\omega_{k}$ on $J^{r} M$ is the $p^{r+1}$-horizontal on $J^{r+1} M$ defined as follows: $\left(D \omega_{k}\right)_{j_{x}^{r+1} s}=\left(p^{r+1}\right)^{*}\left(d\left(\left(j^{r} s\right)^{*} \omega_{k}\right)\right)_{x}$. Hence, we have $D\left(f d x^{i_{1}} \wedge \cdots \wedge d x^{i_{k}}\right)=\left(D_{i} f\right) d x^{i} \wedge d x^{i_{1}} \wedge \cdots \wedge d x^{i_{k}}, \forall f \in C^{\infty}\left(J^{r} M\right)$.

If $\Phi_{t}: M \rightarrow M$ is the flow of a $p$-projectable vector field $X \in \mathfrak{X}(M)$, then $\left(\Phi_{t}\right)^{r}: J^{r} M \rightarrow J^{r} M$ is the flow of a vector field on $J^{r} M$, denoted by $X^{(r)}$ and called the infinitesimal contact transformation of order $r$ associated to $X$. The map $X \mapsto X^{(r)}$ is an injection of Lie algebras. If $X=u^{i} \partial / \partial x^{i}+v^{\alpha} \partial / \partial y^{\alpha}$, where $u^{i} \in C^{\infty}(N)$ and $v^{\alpha} \in C^{\infty}(M)$, then (e.g., see [16, 19, 21]) we have

$$
\begin{gathered}
X^{(r)}=u^{i} \frac{\partial}{\partial x^{i}}+\sum_{|I|=0}^{r} v_{I}^{\alpha} \frac{\partial}{\partial y_{I}^{\alpha}}, \quad v_{0}^{\alpha}=v^{\alpha}, \\
v_{I}^{\alpha}=D^{I} v^{\alpha}-\sum_{J<I}\left(\begin{array}{l}
I \\
J
\end{array}\right) \frac{\partial^{|I-J|} u^{i}}{\partial x^{I-J}} y_{J+(i)}^{\alpha}, \quad 1 \leq|I| \leq r .
\end{gathered}
$$

2.3 - Lagrangian densities.

A Lagrangian density of order $r$ on $p: M \rightarrow N$ is a $p^{r}$-horizontal differential $n$-form $\Lambda$ on $p^{r}: J^{r} M \rightarrow N$. If $N$ is orientable and oriented by a volume form $\boldsymbol{v}$, then every Lagrangian density can be written in a unique way as $\Lambda=L\left(p^{r}\right)^{*} \boldsymbol{v}$, where $L \in C^{\infty}\left(J^{r} M\right)$. Usually, we shall simply write $\Lambda=L \boldsymbol{v}$. In this case, we confine ourselves to consider coordinate systems $\left(x^{i}\right)$ on $N$ adapted to $\boldsymbol{v}$, as shown in (2).

\section{Ehresmann connections.}

An Ehresmann (or non-linear) connection of order $r$ on $p: M \rightarrow N$ is a differential 1-form $\gamma^{r}$ on $J^{r-1} M$ with values in the vertical sub-bundle $V\left(p^{r-1}\right)$ such that $\gamma^{r}(X)=X$ for every $X \in V\left(p^{r-1}\right)$ (e.g., see [14, 20, 21]). Once a connection $\gamma^{r}$ is given, we have a decomposition of vector bundles $T\left(J^{r-1} M\right)=V\left(p^{r-1}\right) \oplus \operatorname{ker} \gamma^{r}$, where $\operatorname{ker} \gamma^{r}$ is called the horizontal subbundle determined by $\gamma^{r}$. In the coordinate system on $J^{r-1} M$ induced from a fibred coordinate system $\left(x^{j}, y^{\alpha}\right)$ for $p$, an Ehresmann connection can be written as

$$
\gamma^{r}=\sum_{|I|=0}^{r-1}\left(d y_{I}^{\alpha}+\gamma_{I j}^{\alpha} d x^{j}\right) \otimes \frac{\partial}{\partial y_{I}^{\alpha}}, \quad \gamma_{I j}^{\alpha} \in C^{\infty}\left(J^{r-1} M\right) .
$$


There is a bijective correspondence between the $r$-th order connections $\gamma^{r}$ on $p: M \rightarrow N$ and the linear sections $\tilde{\gamma}^{r}:\left(p^{r-1}\right)^{*} T N \rightarrow T\left(J^{r-1} M\right)$ of the homomorphism $\left(p^{r-1}\right)_{*}: T\left(J^{r-1} M\right) \rightarrow\left(p^{r-1}\right)^{*} T N$ given by the following formula: $X=\gamma^{r}(X)+\tilde{\gamma}^{r}\left(j_{x}^{r-1} s,\left(p^{r-1}\right)_{*} X\right), \forall X \in T_{j_{x}^{r-1} s}\left(J^{r-1} M\right)$.

The sections of the affine bundle $p^{r, r-1}: J^{r} M \rightarrow J^{r-1} M$ are usually called $r$-th order jet fields (e.g., see [21, §4.6, §5.4]). If $\sigma: J^{r-1} M \rightarrow J^{r} M$ is such a field and $\vartheta^{r}=\sum_{|I|<r-1} \vartheta_{I}^{\alpha} \otimes \partial / \partial y_{I}^{\alpha}$ is the structure form on $J^{r} M$ (see the formula (10) and [16]), then $\gamma_{\sigma}^{r}=\sigma^{*} \vartheta^{r}$ is a connection of order $r$. As an affine bundle admits global sections (e.g., see [11, I, Theorem 5.7]), this proves that every fibred manifold admits connections of any order. Furthermore, the map $\sigma \mapsto \gamma_{\sigma}^{r}=\sigma^{*} \vartheta^{r}$ is an injection from jet fields into connections. In fact, with the same notations as in (13), we have

$$
\begin{array}{ll}
\gamma_{I j}^{\alpha}=-y_{I+(j)}^{\alpha}, & 0 \leq|I| \leq r-2, \\
\gamma_{I j}^{\alpha}=-y_{I+(j)}^{\alpha} \circ \sigma, & |I|=r-1 .
\end{array}
$$

For $r=1$ the correspondence $\sigma \mapsto \gamma_{\sigma}^{1}=\sigma^{*} \vartheta^{1}$ is bijective. Hence $\vartheta^{1}$ is the «universal» connection form. Every $r$-th order jet field $\sigma: J^{r-1} M \rightarrow J^{r} M$ can be viewed as a first-order jet field $\varphi \circ \sigma: J^{r-1} M \rightarrow J^{1}\left(J^{r-1} M\right)$ along the projection $p^{r-1}: J^{r-1} M \rightarrow N$, where $\varphi: J^{r} M \rightarrow J^{1}\left(J^{r-1} M\right)$ is the natural embedding. The $r$-th order connection $\sigma^{*} \vartheta^{r}$ coincides with the first-order connection induced by $\varphi \circ \sigma$; i.e., $\sigma^{*} \vartheta_{M}^{r}=(\varphi \circ \sigma)^{*} \vartheta_{J^{r-1} M}^{1}$.

Proposition 3.1. Every r-order connection $\gamma^{r}$ on $p: M \rightarrow N$ induces an affine map of affine bundles over $J^{r-1} M$,

$$
h_{\gamma^{r}}: J^{r} M \rightarrow \operatorname{Hom}\left(\left(p^{r-1}\right)^{*} T N, V\left(p^{r-1}\right)\right)
$$

given by $h_{\gamma^{r}}\left(j_{x}^{r} s\right)=\left(\left(j^{r-1} s\right)^{*} \gamma^{r}\right)_{x}$, whose associated linear mapping is the inclusion $S^{r} T^{*} N \otimes_{J^{r-1} M} V(p) \subset T^{*} N \otimes_{J^{r-1} M} V\left(p^{r-1}\right)$. Moreover, if $\gamma_{\sigma}^{r}=\sigma^{*} \vartheta^{r}$ for a jet field $\sigma: J^{r-1} M \rightarrow J^{r} M$, then $\operatorname{im} h_{\gamma^{r}} \subseteq S^{r} T^{*} N \otimes_{J^{r-1} M} V(p)$ and we have $h_{\gamma^{r}}\left(j_{x}^{r} s\right)+\sigma\left(j_{x}^{r-1} s\right)=j_{x}^{r} s$.

Proof. From the formula (13) and the definition of $h_{\gamma^{r}}$ we obtain

$$
h_{\gamma^{r}}\left(j_{x}^{r} s\right)=\sum_{|I|=0}^{r-1}\left(\frac{\partial^{|I|+1}\left(y^{\alpha} \circ s\right)}{\partial x^{I+(j)}}(x)+\gamma_{I j}^{\alpha}\left(j_{x}^{r-1} s\right)\right)\left(d x^{j}\right)_{x} \otimes\left(\frac{\partial}{\partial y_{I}^{\alpha}}\right)_{j_{x}^{r-1} s}
$$

thus proving that $h_{\gamma^{r}}$ is an affine map whose associated linear map is the injection of $S^{r} T^{*} N \otimes_{J^{r-1} M} V(p)$ into $T^{*} N \otimes_{J^{r-1} M} V\left(J^{r-1} M\right)$. Moreover, if 
$\gamma^{r}=\sigma^{*} \vartheta^{r}$, then from the formulas (14) we obtain

$$
h_{\gamma^{r}}=\sum_{|I|=r-1}\left(y_{I+(j)}^{\alpha}-y_{I+(j)}^{\alpha} \circ \sigma \circ p^{r, r-1}\right) d x^{j} \otimes \frac{\partial}{\partial y_{I}^{\alpha}},
$$

and the result follows.

The action of the group Aut $(p)$ on the space of $r$-th order connections is defined by the formula $\Phi \cdot \gamma^{r}=\left(\Phi^{r-1}\right)_{*} \circ \gamma^{r} \circ\left(\Phi^{r-1}\right)_{*}^{-1}, \forall \Phi \in \operatorname{Aut}(p)$. As $\Phi^{r-1}: J^{r-1} M \rightarrow J^{r-1} M$ is a morphism of fibred manifolds over $N,\left(\Phi^{r-1}\right)_{*}$ transforms the vertical sub-bundle $V\left(p^{r-1}\right)$ into itself; hence the previous definition makes sense.

\section{Diffeomorphism invariance on linear frame bundles.}

Let $\pi: F N \rightarrow N$ be the bundle of linear frames of $N$. A Lagrangian density $\Lambda$ defined on $J^{r}(F N)$ is said to be Diff $N$-invariant- or even, invariant under diffeomorphisms-(resp. $\mathfrak{X}(N)$-invariant) if the following equation holds: $\left(\tilde{\phi}^{r}\right)^{*} \Lambda=\Lambda, \forall \phi \in \operatorname{Diff} N$ (resp. $L_{\tilde{X}^{(r)}} \Lambda=0, \forall X \in \mathfrak{X}(N)$ ), where $\tilde{\phi}$ (resp. $\tilde{X} \in \mathfrak{X}(F N)$ ) is the natural lift of $\phi$ (resp. $X)$ to $F N$ ([11, VI, $\S 1, \S 2]$ ), and $\tilde{X}^{(r)}$ denotes the $r$-th jet prolongation of $\tilde{X}$, as introduced in Section 2.2. We can write

$$
\Lambda=\mathcal{L} \theta^{1} \wedge \cdots \wedge \theta^{n},
$$

where $\theta=\left(\theta^{1}, \ldots, \theta^{n}\right)$ is the canonical 1-form on $F N$ ([11, III, $\S 2$, p. 118]), and $\mathcal{L} \in C^{\infty}\left(J^{r}(F N)\right)$ is called the «canonical Lagrangian» associated to $\Lambda$. A density $\Lambda$ is Diff $N$-invariant (resp. $\mathfrak{X}(N)$-invariant) if and only if $\mathcal{L} \circ \tilde{\phi}^{r}=\mathcal{L}, \forall \phi \in \operatorname{Diff} N$ (resp. $\tilde{X}^{(r)} \mathcal{L}=0, \forall X \in \mathfrak{X}(N)$ ), as $\theta$ is Diff $N$-invariant and hence, $\mathfrak{X}(N)$-invariant. The problem of determining invariant Lagrangian densities is thus reduced to that of determining invariant Lagrangian functions. Moreover, Diff $N$-invariance implies $\mathfrak{X}(N)$-invariance and both notions are equivalent except when $N$ is orientable and admits an orientation-reversing diffeomorphism onto itself (see $[18, \S 2.1]$ ). Because of this, we consider only $\mathfrak{X}(N)$-invariance.

Each coordinate system $\left(x^{i}\right)$ on an open domain $U \subseteq N$ induces a coordinate system $\left(x^{i}, x_{j}^{i}\right)$ on $F U=\pi^{-1}(U)$ by setting

$$
u=\left(\left(\partial / \partial x^{1}\right)_{x}, \ldots,\left(\partial / \partial x^{n}\right)_{x}\right) \cdot\left(\left(x_{j}^{i}(u)\right), \quad x=\pi(u),\right.
$$


and also, according to Section 2.1, a coordinate system $\left(x^{i}, x_{j}^{i}, x_{j,\left(k_{1} \ldots k_{q}\right)}^{i}\right)$, $1 \leq q \leq r, k_{1} \leq \ldots \leq k_{q}$, on $J^{r}(F U)$ by

$$
x_{j,\left(k_{1} \ldots k_{q}\right)}^{i}\left(j_{x}^{r} s\right)=\left(\partial^{q}\left(x_{j}^{i} \circ s\right) / \partial x^{k_{1}} \ldots \partial x^{k_{q}}\right)(x) .
$$

From the local expression of the canonical 1-form

$$
\theta^{i}=y_{j}^{i} d x^{j}, \quad\left(y_{j}^{i}\right)=\left(x_{j}^{i}\right)^{-1},
$$

we deduce that the relation between $L$ and $\mathcal{L}$ in the Lagrangian density $\Lambda=L \boldsymbol{v}=\mathcal{L} \theta^{1} \wedge \cdots \wedge \theta^{n}$ is $L=\mathcal{L} \operatorname{det}\left(y_{b}^{a}\right)$, or equivalently, $\mathcal{L}=L \operatorname{det}\left(x_{b}^{a}\right)$.

Every $\mathfrak{X}(N)$-invariant Lagrangian on $J^{1}(F N)$ can be written as a differentiable function of the following $\frac{1}{2} n^{2}(n-1)$ Lagrangians:

$$
\mathcal{L}_{a b}^{c}=\left(x_{a}^{d} x_{b, d}^{e}-x_{b}^{d} x_{a, d}^{e}\right) y_{e}^{c}, \quad a<b,
$$

and every $\mathfrak{X}(N)$-invariant second-order Lagrangian on $J^{2}(F N)$ can be written as a differentiable function of the

$$
\frac{1}{2} n^{2}(n-1)+\frac{1}{6} n^{2}(n-1)(n+1)=\frac{1}{6} n^{2}(n-1)(2 n+5)
$$

Lagrangians: $\mathcal{L}_{a b}^{c}, a<b ; \mathcal{L}_{a b, d}^{c}, a<b, a \leq d$, where $\mathcal{L}_{a b, d}^{c}$ is given as follows:

$$
\begin{aligned}
\mathcal{L}_{a b, d}^{c}= & x_{d}^{r} D_{r}\left(\mathcal{L}_{a b}^{c}\right) \\
= & x_{d}^{r}\left(x_{a, r}^{u} x_{b, u}^{e}-x_{b, r}^{u} x_{a, u}^{e}\right) y_{e}^{c}+x_{d}^{r}\left(x_{a}^{u} x_{b,(r u)}^{e}-x_{b}^{u} x_{a,(r u)}^{e}\right) y_{e}^{c} \\
& -x_{d}^{r} x_{q, r}^{p}\left(x_{a}^{u} x_{b, u}^{e}-x_{b}^{u} x_{a, u}^{e}\right) y_{p}^{c} y_{e}^{q} .
\end{aligned}
$$

The geometric meaning of such functions is the following. If $s: U \rightarrow F N$ is the section induced by a linear frame $\left(X_{1}, \ldots, X_{n}\right)$ on an open neighbourhood of a point $x \in N$, we denote by $\nabla^{s}$ the only linear connection on $F U$ that parallelizes each vector field $X_{i}$; i.e., $\left(\nabla^{s}\right)_{X_{j}} X_{i}=0$, for $i, j=1, \ldots, n$. Then, we have

$$
\begin{aligned}
\mathcal{L}_{a b}^{c}\left(j_{x}^{1} s\right) & =-\omega^{c}\left(\operatorname{Tor}_{\nabla^{s}}\left(X_{a}, X_{b}\right)\right)(x), \\
\mathcal{L}_{a b, d}^{c}\left(j_{x}^{2} s\right) & =-\omega^{c}\left(\nabla^{s} \operatorname{Tor}_{\nabla^{s}}\right)\left(X_{d}, X_{a}, X_{b}\right)(x),
\end{aligned}
$$

where $\left(\omega^{1}, \ldots, \omega^{n}\right)$ denotes the dual coframe. Moreover, the inequality $a \leq d$ is due to the constraints imposed by Bianchi's first identity for the linear connection $\nabla^{s}$; namely, $\mathcal{L}_{a b}^{h} \mathcal{L}_{h d}^{c}+\mathcal{L}_{b d}^{h} \mathcal{L}_{h a}^{c}+\mathcal{L}_{d a}^{h} \mathcal{L}_{h b}^{c}=\mathcal{L}_{b d, a}^{c}+\mathcal{L}_{d a, b}^{c}+\mathcal{L}_{a b, d}^{c}$. For the details of these facts, see $[8,18]$. 


\section{First-order covariant Hamiltonians.}

\section{1 - Covariant Hamiltonians on the bundle of connections.}

First of all, we introduce some basic notations. For further details we refer the reader to [3, 6]. Let $\pi: P \rightarrow N$ be a principal $G$-bundle. Connections on $P$ are identified to the splittings of the sequence of vector bundles over $N$ (cfr. [1, 5, 7, 12]), $0 \rightarrow$ ad $P \rightarrow T_{G} P \stackrel{\pi_{*}}{\longrightarrow} T N \rightarrow 0$, where ad $P \rightarrow N$ is the adjoint bundle, i.e., the bundle associated with $P$ and the adjoint representation of $G$ on its Lie algebra g, and $T_{G} P=(T P) / G$. Hence ad $P \cong V(\pi) / G$. The sections of the quotient bundle $T_{G} P$ are identified to the $G$-invariant vector fields on $P$, and the sections of ad $P$ are identified to the gauge algebra (e.g., see [10]), that is, $\Gamma\left(N, T_{G} P\right)=\operatorname{Aut} P$, $\Gamma(N, \operatorname{ad} P)=\operatorname{gau} P$. We think of gau $P$ as being the «Lie algebra» of the gauge group Gau $P$ (cfr. [2, 5, 7, 10]).

Consequently, there exists a bundle $p: C(P) \rightarrow N$ whose global sections can be identified with the connections on $P$ (e.g., see [1, 5, 7, 12]). This bundle is affine and modelled over $T^{*} N \otimes \operatorname{ad} P$. We denote by $s_{\Gamma}: N \rightarrow C(P)$ the section of the bundle of connections induced tautologically by $\Gamma$.

Let $U \subseteq M$ be an open subset such that $\pi^{-1}(U) \cong U \times G$. For every $B \in \mathfrak{g}$ there is a flow of gauge transformations $\varphi_{t}^{B}: U \times G \rightarrow U \times G$ given by $\varphi_{t}^{B}(x, g)=(x, \exp (t B) \cdot g)$. The infinitesimal generator of $\varphi_{t}^{B}$ is denoted by $\bar{B}$, which is an infinitesimal gauge transformation on $\pi^{-1}(U)$.

Let $\tilde{B}=\bar{B}(\bmod G)$ be the orbit of $\bar{B}$ in ad $P$. If $\left(B_{\alpha}\right)$ is a basis of $\mathrm{g}$, then $\left(\tilde{B}_{\alpha}\right)$ is a basis of $\Gamma(U, \operatorname{ad} P)=\operatorname{gau} \pi^{-1}(U)$. Accordingly, every gauge field $X \in$ gau $P$ can be written as

$$
X=g^{\alpha} \tilde{B}_{\alpha}, \quad g^{\alpha} \in C^{\infty}(U) .
$$

Coordinates on $C(P)$ are introduced as follows: Let $\left(U, x^{i}\right)$ be a coordinate domain in $N$ such that $\pi^{-1}(U) \cong U \times G$. We can define functions $A_{j}^{\alpha}: p^{-1}(U) \rightarrow \mathbb{R}$, by setting

$$
\left(\partial / \partial x^{j}\right)_{x}^{h\left(\Gamma_{x}\right)}=\left(\partial / \partial x^{j}\right)_{x}-A_{j}^{\alpha}\left(\Gamma_{x}\right)\left(\tilde{B}_{\alpha}\right)_{x},
$$

for every connection $\Gamma_{x}$ at a point $x \in U$, where $h\left(\Gamma_{x}\right)$ denotes the horizontal lift with respect to $\Gamma_{x}$. The functions $\left(x^{j}, A_{j}^{\alpha}\right)$ constitute a coordinate system on $p^{-1}(U)$. According to the general notations introduced in Section 2.1 , we denote by $\left(x^{j}, A_{j}^{\alpha} ; A_{j, k}^{\alpha}\right)$ the induced coordinate system on $J^{1} C(P)$. 
Each gauge transformation $\Phi \in$ GauP acts on connections by pulling connection forms back (see [11, II, Proposition 6.2-(b)]): $\Gamma^{\prime}=\Phi \cdot \Gamma$, where $\omega_{\Gamma^{\prime}}=\left(\Phi^{-1}\right)^{*} \omega_{\Gamma}$. Hence a unique diffeomorphism $\Phi_{C}: C(P) \rightarrow C(P)$ exists such that $\Phi_{C} \circ s_{\Gamma}=s_{\Phi \cdot \Gamma}$, for every connection $\Gamma$ on $P$.

If $\Phi_{t}$ is the flow of a gauge field $X \in \operatorname{gau} P$, then $\left(\Phi_{t}\right)_{C}$ is a flow on $C(P)$ and the corresponding infinitesimal generator is denoted by $X_{C}$; its local expression is (see [3, 6]),

$$
X_{C}=\left(c_{\alpha \gamma}^{\beta} g^{\alpha} A_{i}^{\gamma}-\frac{\partial g^{\beta}}{\partial x^{i}}\right) \frac{\partial}{\partial A_{i}^{\beta}},
$$

where $c_{\beta \gamma}^{\alpha}$ are the structural constants, $\left[B_{\beta}, B_{\gamma}\right]=c_{\beta \gamma}^{\alpha} B_{\alpha}$, and $X$ is given by the formula (21).

According to the general equation (7), a Lagrangian $L \in C^{\infty}\left(J^{1} C(P)\right)$ is gauge invariant if $X_{C}^{(1)} L=0, \forall X \in$ gau $P$. Since $X_{C}^{(1)}$ is $p^{1}$-vertical, we have $L_{X_{C}^{(1)}}(L \boldsymbol{v})=X_{C}^{(1)}(L) \boldsymbol{v}$. Hence the gauge invariance of the density $L \boldsymbol{v}$ is reduced to that of the Lagrangian function.

The classification of gauge invariant Lagrangian densities is given by the geometric formulation of Utiyama's theorem ([2, 7, 23]), which establishes that a Lagrangian $L$ is gauge invariant if and only if there exists a smooth function $\bar{L}: \wedge^{2} T^{*} N \otimes$ ad $P \rightarrow \mathbb{R}$, which in turn must also be invariant under the natural action of the adjoint representation on $\wedge^{2} T^{*} N \otimes$ ad $P$, such that $L=\bar{L} \circ \Omega$, where $\Omega: J^{1} C(P) \rightarrow \wedge^{2} T^{*} N \otimes \operatorname{ad} P$ is the curvature mapping, i.e., $\Omega\left(j_{x}^{1} s_{\Gamma}\right)=\left(\Omega_{\Gamma}\right)_{x}$.

Let $R_{j k}^{\alpha}, j<k$, be the coordinate system induced by $\left(x^{j}\right)$ and $\left(B_{\alpha}\right)$ on the curvature bundle; i.e., $\eta_{2}=\sum_{j<k} R_{j k}^{\alpha}\left(\eta_{2}\right)\left(d x^{j}\right)_{x} \wedge\left(d x^{k}\right)_{x} \otimes\left(\tilde{B}_{\alpha}\right)_{x}$, for every 2covector $\eta_{2} \in \wedge^{2} T_{x}^{*} N \otimes(\operatorname{ad} P)_{x}$. Then, the equations of the curvature mapping are (e.g., see [6]),

$$
R_{j k}^{\alpha} \circ \Omega=A_{j, k}^{\alpha}-A_{k, j}^{\alpha}-c_{\beta \gamma}^{\alpha} A_{j}^{\beta} A_{k}^{\gamma} .
$$

From the general formula (13) we obtain the local expression of a firstorder Ehresmann connection $\gamma^{1}$ on $C(P)$ in the coordinates $\left(x^{i}, A_{i}^{\alpha}\right)$ introduced in the formula (22); precisely,

$$
\gamma^{1}=\left(d A_{j}^{\alpha}+\gamma_{j k}^{\alpha} d x^{k}\right) \otimes \frac{\partial}{\partial A_{j}^{\alpha}}, \quad \gamma_{j k}^{\alpha} \in C^{\infty}(C(P)) .
$$

If $p: M \rightarrow N$ is an affine bundle modelled over a vector bundle $q: W \rightarrow N$, then we have an isomorphism $p^{*} W \rightarrow V(p)$ of vector bundles over $M$, that maps the pair $(y, w) \in M \times q^{-1}(p(y))$ onto the tangent vector $\chi(y, w) \in V_{y}(p)$ given by $\chi(y, w)(f)=\lim _{t \rightarrow 0} \frac{1}{t}(f(y+t w)-f(y)), \forall f \in C^{\infty}(M)$. 
As $p: C(P) \rightarrow N$ is an affine bundle modelled over $T^{*} N \otimes$ ad $P$, a firstorder Ehresmann connection $\gamma^{1}$ on $C(P)$ can be viewed as a 1-form on $C(P)$ with values in the vector bundle $p^{*}\left(T^{*} N \otimes\right.$ ad $\left.P\right)$. By taking its horizontal part, we conclude that hor $\gamma^{1}$ defines a section of the vector bundle $\left(p^{1}\right)^{*}\left(T^{*} N \otimes T^{*} N \otimes \operatorname{ad} P\right)$, or even a morphism of fibred manifolds over $N$,

$$
\text { hor } \gamma^{1}: J^{1} C(P) \rightarrow T^{*} N \otimes T^{*} N \otimes \operatorname{ad} P .
$$

LEMma 5.1. With the same notations as above, the section corresponding to $d x^{j} \otimes \tilde{B}_{\alpha}$ in the isomorphism $p^{*}\left(T^{*} N \otimes \operatorname{ad} P\right) \rightarrow V(p)$, is $-\partial / \partial A_{j}^{\alpha}$.

Proof. According to the formula (22), for every $\Gamma_{x} \in C(P)$, we have

$$
A_{i}^{\beta}\left(\Gamma_{x}+t\left(d x^{j} \otimes \tilde{B}_{\alpha}\right)_{x}\right)=A_{i}^{\beta}\left(\Gamma_{x}\right)-t \delta_{i}^{j} \delta_{\alpha}^{\beta} .
$$

Hence $\chi\left(\Gamma_{x},\left(d x^{j} \otimes \tilde{B}_{\alpha}\right)_{x}\right)=-\left(\partial / \partial A_{j}^{\alpha}\right)_{\Gamma_{x}}$.

Theorem 5.2. Let $\pi: P \rightarrow N$ be a principal G-bundle. If $\gamma^{1}$ is a firstorder Ehresmann connection on the bundle of connections $p: C(P) \rightarrow N$ of $P$ satisfying the following equation:

$$
\operatorname{alt}\left(\operatorname{hor} \gamma^{1}\right)=\Omega,
$$

$\Omega$ being the curvature mapping and alt: $\otimes^{2} T^{*} N \otimes \operatorname{ad} P \rightarrow \wedge^{2} T^{*} N \otimes \operatorname{ad} P$ the antisymmetrization operator, then the covariant Hamiltonian $L^{\gamma^{1}} \boldsymbol{v}$ of every gauge invariant Lagrangian $L$ on $J^{1} C(P)$ is gauge invariant.

For every principal bundle $P$, first-order Ehresmann connections globally defined on $C(P)$ satisfying the equation (26), always exist.

Proof. According to [6, Theorem 1-(2)], if $\gamma^{1}$ satisfies the following equation:

$$
\Delta_{j k}^{\alpha}=\gamma_{j k}^{\alpha}-\gamma_{k j}^{\alpha}=-c_{\beta \gamma}^{\alpha} A_{j}^{\beta} A_{k}^{\gamma}, \quad j<k,
$$

then $L^{\gamma^{1}}$ is gauge invariant for every gauge invariant Lagrangian $L$ on $J^{1} C(P)$. Moreover, by applying Lemma 5.1 to the formula (25) we obtain

$$
\gamma^{1}=-\left(d A_{j}^{\alpha}+\gamma_{j k}^{\alpha} d x^{k}\right) \otimes d x^{j} \otimes \tilde{B}_{\alpha},
$$

and by taking the horizontal part, hor $\gamma^{1}=-\left(A_{j, k}^{\alpha}+\gamma_{j k}^{\alpha}\right) d x^{k} \otimes d x^{j} \otimes \tilde{B}_{\alpha}$. Hence alt $\left(\right.$ hor $\left.\gamma^{1}\right)=\sum_{j<k}\left(A_{j, k}^{\alpha}-A_{k, j}^{\alpha}+\Delta_{j, k}^{\alpha}\right) d x^{j} \wedge d x^{k} \otimes \tilde{B}_{\alpha}$, and the formula (26) in the statement follows from the equations of the curvature mapping in the formula (24) whenever $\gamma^{1}$ satisfies the equation (27) above. 
Finally, as remarked in Section 3, there is a one-to-one correspondence between first-order Ehresmann connections and first-order jet fields given by $\gamma_{\sigma}^{1}=\sigma^{*} \vartheta^{1}$. The sections $\sigma$ for which the corresponding Ehresmann connection $\gamma_{\sigma}^{1}$ satisfies the equation (26), are the smooth sections of an affine sub-bundle of the bundle $p^{10}: J^{1} C(P) \rightarrow C(P)$, which is modelled over the vector sub-bundle $p^{*}\left(S^{2} T^{*} N \otimes\right.$ ad $\left.P\right) \subset p^{*}\left(\otimes^{2} T^{*} N \otimes \operatorname{ad} P\right)$, thus proving the last part of the statement.

If the centre of $g$ is trivial, then the equation (26) in Theorem 5.2 is also necessary for the covariant Hamiltonian of every gauge invariant Lagrangian on $J^{1} C(P)$ to be gauge invariant; see [6, Theorem 1-(2)]. Moreover, since $X_{C}$ is $p$-vertical, $L_{X_{C}} \gamma^{1}$ defines a section of the vector bundle $p^{*}\left(\otimes^{2} T^{*} N \otimes \operatorname{ad} P\right)$, as remarked in the proof of Proposition 1.1, and the antisymmetric part of this section vanishes for all gauge field $X$, if and only if $\gamma^{1}$ solves the question (Q); see [6, Proposition 6] for the details of the proof.

Although Theorem 5.2 guarantees the existence of Ehresmann connections solving the question (Q) for the bundle of connections of a principal bundle, we remark on the fact that there is no gauge invariant Ehresmann connection on $C(P)$. In fact, once a point $\Gamma_{x} \in C(P)$ and a coordinate system $\left(x^{i}\right)$ centred at $x$ have been fixed, from the formulas (21), (23) we conclude that the vector field $X_{C}$ for $g^{\alpha}(x)=0$, $\left(\partial g^{\alpha} / \partial x^{i}\right)(x)=0, \forall \alpha, i$, but $\left(\partial^{2} g^{1} / \partial\left(x^{1}\right)^{2}\right)(x) \neq 0$, satisfies the conditions of Proposition 1.2.

\section{2 - Covariant Hamiltonians on linear frame bundles.}

Let $\left(U ; x^{i}\right)$ be a coordinate domain in $N$. According to (13), on the induced coordinate system $\left(x^{i}, x_{j}^{i}\right.$ ) for $\pi: F N \rightarrow N$ (see (16) above), an Ehresmann connection $\gamma^{1}$ on $F N$ can be written as $\gamma^{1}=\left(d x_{j}^{i}+\gamma_{j k}^{i} d x^{k}\right) \otimes \partial / \partial x_{j}^{i}$, with $\gamma_{j k}^{i} \in C^{\infty}(F N)$.

Let $\Lambda^{\gamma^{1}}$ be the covariant Hamiltonian associated to a Lagrangian density $\Lambda$ on $J^{1}(F N)$ with respect to $\gamma^{1}$. As the covariant Hamiltonian $\Lambda^{\gamma^{1}}$ is a Lagrangian density, it also admits a canonical Lagrangian $\mathcal{L}^{\gamma^{1}} \in C^{\infty}\left(J^{1}(F N)\right)$ defined, according to the formula (15), by $\Lambda^{\gamma^{1}}=\mathcal{L}^{\gamma^{1}} \theta^{1} \wedge \cdots \wedge \theta^{n}$, where

$$
\mathcal{L}^{\gamma^{1}}=\left(\gamma_{j k}^{i}+x_{j, k}^{i}\right) \frac{\partial \mathcal{L}}{\partial x_{j, k}^{i}}-\mathcal{L}, \quad \gamma_{j k}^{i} \in C^{\infty}(F N)
$$


LEMma 5.3. The covariant Hamiltonian $\Lambda^{\gamma^{1}}$ of every $\mathfrak{X}(N)$-invariant Lagrangian $\mathcal{L}$ is $\mathfrak{X}(N)$-invariant if and only if all the functions $\left(\mathcal{L}_{a b}^{c}\right)^{\gamma^{1}}$, $a<b$, are $\mathfrak{X}(N)$-invariant.

Proof. If the covariant Hamiltonian $\Lambda^{\gamma^{1}}$ of every $\mathfrak{X}(N)$-invariant Lagrangian $\mathcal{L}$ is $\mathfrak{X}(N)$-invariant, then all the functions $\left(\mathcal{L}_{a b}^{c}\right)^{\gamma^{1}}, a<b$, are, in particular, $\mathfrak{X}(N)$-invariant. Next, we prove the converse. If $X=u^{i} \partial / \partial x^{i}$, $u^{i} \in C^{\infty}(N)$, then

$$
\tilde{X}=u^{i} \frac{\partial}{\partial x^{i}}+x_{j}^{l} \frac{\partial u^{i}}{\partial x^{l}} \frac{\partial}{\partial x_{j}^{i}},
$$

and from the formulas (11), (12) above for $r=1$, we obtain

$$
\left\{\begin{aligned}
\tilde{X}^{(1)} & =u^{i} \frac{\partial}{\partial x^{i}}+v_{j}^{i} \frac{\partial}{\partial x_{j}^{i}}+v_{j k}^{i} \frac{\partial}{\partial x_{j, k}^{i}}, \\
v_{j}^{i} & =\frac{\partial u^{i}}{\partial x^{l}} x_{j}^{l}, \\
v_{j k}^{i} & =\frac{\partial u^{i}}{\partial x^{r}} x_{j, k}^{r}-\frac{\partial u^{r}}{\partial x^{k}} x_{j, r}^{i}+\frac{\partial^{2} u^{i}}{\partial x^{k} \partial x^{r}} x_{j}^{r} .
\end{aligned}\right.
$$

From the formulas (28), (30), we obtain, after some computations,

$$
\tilde{X}^{(1)}\left(\mathcal{L}^{\gamma^{1}}\right)=C_{j k}^{i}(\tilde{X}) \frac{\partial \mathcal{L}}{\partial x_{j, k}^{i}},
$$

where

$$
C_{j k}^{i}(\tilde{X})=\tilde{X} \gamma_{j k}^{i}+v_{j k}^{i}-\left(\gamma_{s t}^{r}+x_{s, t}^{r}\right) \frac{\partial v_{j k}^{i}}{\partial x_{s, t}^{r}} .
$$

As mentioned above, every invariant Lagrangian can be written as a smooth function of the Lagrangians $\mathcal{L}_{a b}^{c}$ defined in the formula (18). Accordingly, we can write $\mathcal{L}=\Phi \circ \Pi^{1}$, for a certain function $\Phi \in C^{\infty}\left(\mathbb{R}^{\frac{1}{2} n^{2}(n-1)}\right)$, where $\Pi^{1}: J^{1}(F N) \rightarrow \mathbb{R}^{\frac{1}{n^{2}(n-1)}}$ is the map whose components are the functions $\mathcal{L}_{a b}^{c}$, i.e., $t_{a b}^{c} \circ \Pi^{1}=\mathcal{L}_{a b}^{c}$, where $\left(t_{a b}^{c}\right), a<b$, are the standard coordinates on $\mathbb{R}^{\frac{1}{2} n^{2}(n-1)}$. Hence, substituting

$$
\frac{\partial \mathcal{L}}{\partial x_{j, k}^{i}}=\left(\frac{\partial \Phi}{\partial t_{a b}^{c}} \circ \Pi^{1}\right) \frac{\partial \mathcal{L}_{a b}^{c}}{\partial x_{j, k}^{i}}
$$

into the equation (31), we obtain

$$
\tilde{X}^{(1)}\left(\mathcal{L}^{\gamma^{1}}\right)=\left(\frac{\partial \Phi}{\partial t_{a b}^{c}} \circ \Pi^{1}\right) \tilde{X}^{(1)}\left(\left(\mathcal{L}_{a b}^{c}\right)^{\gamma^{1}}\right),
$$

and the result follows. 
The notion of torsion of an Ehresmann connection $\gamma^{1}$ on $F N$, can be introduced by imitating that of a linear connection; e.g., see [11, III, § 2]. Let $B(v)$ be the standard horizontal vector field associated to $v \in \mathbb{R}^{n}$ (see [11, III, §2]) with respect to $\gamma^{1}$; precisely, $B(v)$ is the only $\gamma^{1}$-horizontal vector field on $F N$ such that $\pi_{*}\left(B(v)_{u}\right)=u(v)$ for every $u \in F_{x} N$, where, here, the linear frame $u$ is considered to be a linear isomorphism $u: \mathbb{R}^{n} \rightarrow T_{x} N$. Then, we define the torsion of $\gamma^{1}$ as being the map $T^{\gamma^{1}}: F N \rightarrow \wedge^{2}\left(\mathbb{R}^{n}\right)^{*} \otimes \mathbb{R}^{n}$ given by $T^{\gamma^{1}}(u)(v, w)=d \theta(B(v), B(w))(u), \forall u \in F N, \forall v, w \in \mathbb{R}^{n}$, and $\theta$ is the canonical 1-form on $F N$ introduced in Section 4 above. Let $\left(v_{i}\right)$ be the standard basis of $\mathbb{R}^{n}$ with dual basis $\left(v^{i}\right)$, and let $\left(T^{\gamma^{1}}\right)_{j k}^{i}(u), j<k$, be the components of $T^{\gamma^{1}}(u)$ in such basis, i.e.,

$$
T^{\gamma^{1}}(u)=\sum_{j<k}\left(T^{\gamma^{1}}\right)_{j k}^{i}(u) v^{j} \wedge v^{k} \otimes v_{i}
$$

From the local expression $B\left(v_{a}\right)=x_{a}^{k}\left(\partial / \partial x^{k}-\gamma_{j k}^{i} \partial / \partial x_{j}^{i}\right)$ of the standard horizontal vector field associated to $v_{a}$ and that of $\theta$ in the formula (17), we obtain

$$
\left(T^{\gamma^{1}}\right)_{a b}^{c}=\left(x_{a}^{k} \gamma_{b k}^{i}-x_{b}^{k} \gamma_{a k}^{i}\right) y_{i}^{c}
$$

Theorem 5.4. Let $\gamma^{1}$ be an Ehresmann connection on $\pi: J^{1}(F N) \rightarrow N$. The covariant Hamiltonian $\Lambda^{\gamma^{1}}$ associated with every $\mathfrak{X}(N)$-invariant Lagrangian density $\Lambda$ on $J^{1}(F N)$ is $\mathfrak{X}(N)$-invariant, if and only if the torsion of $\gamma^{1}$ is constant.

Proof. Let $\mathcal{L}^{\gamma^{1}} \in C^{\infty}\left(J^{1}(F N)\right)$ be the canonical Lagrangian associated to the covariant Hamiltonian $\mathcal{1}^{\gamma^{1}}$. We must prove that $\tilde{X}^{(1)}\left(\mathcal{L}^{\gamma^{1}}\right)=0$, for every $X \in \mathfrak{X}(N)$ and every $\mathfrak{X}(N)$-invariant Lagrangian $\mathcal{L}$, if and only if the torsion of $\gamma^{1}$ is constant. As follows from Lemma 5.3, we only need to impose $\tilde{X}^{(1)}\left(\left(\mathcal{L}_{a b}^{c} \gamma^{\gamma^{1}}\right)=0, \forall X \in \mathfrak{X}(N)\right.$, and all indices $a<b, c$. Taking the following identity:

$$
\frac{\partial \mathcal{L}_{a b}^{c}}{\partial x_{j, k}^{i}}=\left(x_{a}^{k} \delta_{b}^{j}-x_{b}^{k} \delta_{a}^{j}\right) y_{i}^{c}
$$

into account, from the formula (33) we obtain

$$
\left(\mathcal{L}_{a b}^{c}\right)^{\gamma^{1}}=\left(\gamma_{j k}^{i}+x_{j, k}^{i}\right) \frac{\partial \mathcal{L}_{a b}^{c}}{\partial x_{j, k}^{i}}-\mathcal{L}_{a b}^{c}=\gamma_{j k}^{i} \frac{\partial \mathcal{L}_{a b}^{c}}{\partial x_{j, k}^{i}}=\left(T^{\gamma^{1}}\right)_{a b}^{c} .
$$

Hence $\tilde{X}^{(1)}\left(\mathcal{L}_{a b}^{c}\right)^{\gamma^{1}}=\tilde{X}\left(T^{\gamma^{1}}\right)_{a b}^{c}$, which allows us to conclude the proof. 
COROLLARY 5.5. Let $\Gamma$ be a linear connection on FN. The covariant Hamiltonian $\Lambda^{\Gamma}$ associated with every $\mathfrak{X}(N)$-invariant Lagrangian density $\Lambda$ on $J^{1}(F N)$ is $\mathfrak{X}(N)$-invariant, if and only if $\Gamma$ is torsion free.

Proof. The torsion of a linear connection is equivariant with respect to the natural actions of $G L(n ; \mathbb{R})$ on $F N$ and on $\wedge^{2}\left(\mathbb{R}^{n}\right)^{*} \otimes \mathbb{R}^{n}$, respectively; that is, $T^{\Gamma}(u \cdot A)=A^{-1} \cdot T^{\Gamma}(u), \forall u \in F N, \forall A \in G L(n ; \mathbb{R})$. Hence, if $T^{\Gamma}$ is constant, then $T^{\Gamma}(u)=T^{\Gamma}(u \cdot A)=A^{-1} \cdot T^{\Gamma}(u), \forall u \in F N, \forall A \in G L(n ; \mathbb{R})$. Accordingly, $T^{\Gamma}(u)$ is $G L(n ; \mathbb{R})$-invariant, so that $T^{\Gamma}(u)$ must vanish.

Let $I_{1}: F N \times \mathfrak{g I}(n, \mathbb{R}) \rightarrow V(\pi)$ be the vector-bundle isomorphism defined by $I_{1}(u, A)=A_{u}^{*}$, where $A^{*}$ stands for the fundamental vector field attached to $A \in \mathfrak{g l}(n, \mathbb{R})$, i.e., the infinitesimal generator of the flow $R_{\exp (t A)}$. If $\left(E_{j}^{i}\right)$ denotes the standard basis of $\mathfrak{g l}(n, \mathbb{R})$, then in the induced coordinate system introduced in the formula (16), we have $I_{1}\left(u, E_{j}^{i}\right)=$ $=x_{i}^{h}(u)\left(\partial / \partial x_{j}^{h}\right)_{u}$, for all $u \in F N$.

We also have an isomorphism $I_{2}: \pi^{*}\left(T^{*} N \otimes T N\right) \rightarrow F N \times \mathfrak{g l}(n, \mathbb{R})$ given by $I_{2}(u, F)=\left(u,\left(a_{j}^{i}\right)\right)$, where $F\left(X_{j}\right)=a_{j}^{i} X_{i}$, and $u=\left(X_{1}, \ldots, X_{n}\right)$. In local coordinates, we obtain $I_{2}\left(u, d x^{b} \otimes \partial / \partial x^{c}\right)=\left(u,\left(a_{j}^{i}\right)\right)$, with $a_{j}^{i}=y_{c}^{i}(u) x_{j}^{b}(u)$.

Below, we need to consider the isomorphism

$$
\left\{\begin{array}{l}
I=I_{2}^{-1} \circ I_{1}^{-1}: V(\pi) \rightarrow \pi^{*}\left(T^{*} N \otimes T N\right), \\
I\left(\frac{\partial}{\partial x_{j}^{i}}\right)=y_{k}^{j} d x^{k} \otimes \frac{\partial}{\partial x^{i}} .
\end{array}\right.
$$

For every $X \in \mathfrak{X}(N)$, we know that $L_{\tilde{X}} \gamma^{1}$ defines a section of the vector bundle $\pi^{*} T^{*} N \otimes V(\pi)$, which can be identified to $\pi^{*}\left(\otimes^{2} T^{*} N \otimes T N\right)$, taking the isomorphism (35) into account.

Proposition 5.6. The covariant Hamiltonian $\Lambda^{\gamma^{1}}$ associated to every $\mathfrak{X}(N)$-invariant Lagrangian density $\Lambda$ on $J^{1}(F N)$ is $\mathfrak{X}(N)$-invariant if and only if $\operatorname{alt}\left(L_{\tilde{X}} \gamma^{1}\right)=0$, for all $X \in \mathfrak{X}(N)$, where alt: $\otimes^{2} T^{*} N \otimes T N \rightarrow$ $\rightarrow \wedge^{2} T^{*} N \otimes T N$ is the antisymmetrization operator.

PRoof. From the equation (29) and the expression of $\gamma^{1}$, taking the isomorphism (35) into account, we obtain $L_{\tilde{X}} \gamma^{1}=C_{j k}^{i}(\tilde{X}) y_{s}^{j} d x^{k} \otimes$ $\otimes d x^{s} \otimes \partial / \partial x^{i}$, where $C_{j k}^{i}(X)$ is given by the formula (32). By setting $X_{j}=x_{j}^{h} \partial / \partial x^{h}$, the previous equation can be rewritten as $L_{\tilde{X}} \gamma^{1}=$ $=x_{a}^{k} C_{b k}^{i}(\tilde{X}) y_{i}^{c} \theta^{a} \otimes \theta^{b} \otimes X_{c}$. From the formula (33), taking the equation $\partial y_{i}^{c} / \partial x_{r}^{h}=-y_{h}^{c} y_{i}^{r}$ and the formula (34) into account, the equation above 
yields

$$
\begin{aligned}
\operatorname{alt}\left(L_{\tilde{X}} \gamma^{1}\right) & =\left(\frac{\partial\left(T^{\gamma^{1}}\right)_{a b}^{c}}{\partial x^{r}} u^{r}+x_{t}^{l} \frac{\partial\left(T^{\gamma^{1}}\right)_{a b}^{c}}{\partial x_{t}^{r}} \frac{\partial u^{r}}{\partial x^{l}}\right) \theta^{a} \wedge \theta^{b} \otimes X_{c} \\
& =\tilde{X}\left(\left(T^{\gamma^{1}}\right)_{a b}^{c}\right) \theta^{a} \wedge \theta^{b} \otimes X_{c} .
\end{aligned}
$$

Hence, from the formula above and Theorem 5.4, the result follows.

Theorem 5.4 guarantees the existence of Ehresmann connections solving the question $(\mathrm{Q})$ for the bundle of first-order linear frames, but there is no $\mathfrak{X}(N)$-invariant Ehresmann connection on $J^{1}(F N)$. In fact, once a coordinate system $\left(x^{i}\right)$ centred at $x=\pi(u)$ adapted to a given point $u \in F N$ (i.e., $x_{j}^{i}(u)=\delta_{j}^{i}$ ) has been fixed, from the formula (29), we conclude that the vector field $\tilde{X}$ for $u^{i}(x)=0,\left(\partial u^{i} / \partial x^{j}\right)(x)=0, \forall i, j$, but $\left(\partial^{2} u^{1} / \partial\left(x^{1}\right)^{2}\right)(x) \neq 0$, satisfies the conditions of Proposition 1.2.

\section{Covariant Hamiltonians for second-order Lagrangians.}

\section{1 - Legendre and Poincaré-Cartan forms.}

The Legendre form of a second-order Lagrangian density $\Lambda=L \boldsymbol{v}$ defined on $p: M \rightarrow N$ is the $V^{*}\left(p^{1}\right)$-valued $p^{3}$-horizontal $(n-1)$-form $\omega_{\Lambda}$ on $J^{3} M$ locally given by $\omega_{\Lambda}=\sum_{|I|=0}^{1}(-1)^{i-1} L_{\alpha}^{i I} \boldsymbol{v}_{i} \otimes d y_{I}^{\alpha}$ (e.g., see [17, 22]), where

$$
\begin{aligned}
L_{\alpha}^{i(j)} & =\frac{1}{2-\delta_{i j}} \frac{\partial L}{\partial y_{(i j)}^{\alpha}}, \\
L_{\alpha}^{i 0} & =\frac{\partial L}{\partial y_{i}^{\alpha}}-\frac{1}{2-\delta_{i j}} D_{j}\left(\frac{\partial L}{\partial y_{(i j)}^{\alpha}}\right) .
\end{aligned}
$$

The Poincaré-Cartan form attached to $\Lambda$ is then defined to be the ordinary $n$ form on $J^{3} M$ given by,

$$
\Theta_{\Lambda}=\left(p^{32}\right)^{*} \vartheta^{2} \wedge \omega_{\Lambda}+\Lambda,
$$

where $\vartheta^{2}$ is the second-order structure form and the exterior product of $\left(p^{32}\right)^{*} \vartheta^{2}$ and the Legendre form, is taken with respect to the pairing induced by duality, $V\left(p^{1}\right) \times{ }_{J^{1} M} V^{*}\left(p^{1}\right) \rightarrow \mathbb{R}$. The most outstanding difference with the first-order case is that the Legendre and Poincaré-Cartan forms associated with a second-order Lagrangian density are generally defined on $J^{3} M$, thus increasing by one the order of the density. 


\section{2 - Hamiltonian attached to a connection.}

Similarly to the first-order case (see [6, 14]), given a second-order Lagrangian density $\Lambda$ on $p: M \rightarrow N$ and a second-order connection $\gamma^{2}$ on $p: M \rightarrow N$, by subtracting $\left(p^{32}\right)^{*} \vartheta^{2}$ from $\left(p^{31}\right)^{*} \gamma^{2}$ we obtain a $p^{3}$-horizontal form, and we can define the corresponding covariant Hamiltonian to be the Lagrangian density of third order $\Lambda^{\gamma^{2}}=\left(\left(p^{31}\right)^{*} \gamma^{2}-\left(p^{32}\right)^{*} \vartheta^{2}\right) \wedge \omega_{\Lambda}-\Lambda$. Expanding on the right-hand side above and taking the formula (38) into account, we obtain the decomposition of the Poincaré-Cartan form that generalizes the formula (5) to second-order Lagrangian densities; precisely, $\Theta_{\Lambda}=\left(p^{31}\right)^{*} \gamma^{2} \wedge$ $\wedge \omega_{\Lambda}-\Lambda^{\gamma^{2}}$. With the same notations as in the formulas (13), (36), (37) we have

$$
L^{\gamma^{2}}=\left(\gamma_{i}^{\alpha}+y_{i}^{\alpha}\right) L_{\alpha}^{i 0}+\left(\gamma_{h i}^{\alpha}+y_{(h i)}^{\alpha}\right) L_{\alpha}^{i(h)}-L .
$$

Because of the formula (37) the covariant Hamiltonian $L^{\gamma^{2}}$ is generally defined on $J^{3} M$. Nevertheless, in view of the applications, we confine ourselves to consider second-order covariant Hamiltonians only. We therefore characterize those second-order Lagrangians whose covariant Hamiltonians are also of second-order, whichever it is the second-order connection $\gamma^{2}$ under consideration.

Looking at the equations (36), (37), we conclude that $L^{\gamma^{2}}$ is defined on $J^{2} M$ if and only if, for every multi-index $I,|I|=3$, and every pair of indices $\alpha, \beta$ we have $\partial L_{\alpha 0}^{i} / \partial y_{I}^{\beta}=0$. Letting $I=(a b c), a \leq b \leq c$, we see that the previous condition is equivalent to the following:

$$
\frac{1}{2-\delta_{i b}} \frac{\partial^{2} L}{\partial y_{(a c)}^{\beta} \partial y_{(i b)}^{\alpha}}+\frac{1}{2-\delta_{i a}} \frac{\partial^{2} L}{\partial y_{(b c)}^{\beta} \partial y_{(i a)}^{\alpha}}+\frac{1}{2-\delta_{i c}} \frac{\partial^{2} L}{\partial y_{(a b)}^{\beta} \partial y_{(i c)}^{\alpha}}=0
$$

We remark on the fact that if the equation (40) holds, then the PoincaréCartan form (38)-initially defined on $J^{3} M$-also projects onto $J^{2} M$.

\section{Second-order Lagrangians on linear frames.}

In the case of the bundle of linear frames $\pi: F N \rightarrow N$ the equation (40) becomes

$$
\begin{aligned}
& \frac{1}{2-\delta_{i b}} \frac{\partial^{2} L}{\partial x_{q,(a c)}^{p} \partial x_{m,(i b)}^{l}} \\
& \quad+\frac{1}{2-\delta_{i a}} \frac{\partial^{2} L}{\partial x_{q,(b c)}^{p} \partial x_{m,(i a)}^{l}}+\frac{1}{2-\delta_{i c}} \frac{\partial^{2} L}{\partial x_{q,(a b)}^{p} \partial x_{m,(i c)}^{l}}=0
\end{aligned}
$$

for all systems of indices $i, l, m, p, q, a \leq b \leq c$. 
Let $\widetilde{S}_{N}$ denote the class of Lagrangians $L \in C^{\infty}\left(J^{2}(F N)\right)$ that satisfy the equations (41).

Let $\gamma^{2}$ be a second-order Ehresmann connection on $\pi: F N \rightarrow N$. According to (13), locally we have

$$
\left\{\begin{array}{l}
\gamma^{2}=\left(d x_{j}^{i}+\gamma_{j k}^{i} d x^{k}\right) \otimes \frac{\partial}{\partial x_{j}^{i}}+\left(d x_{j, h}^{i}+\gamma_{j h k}^{i} d x^{k}\right) \otimes \frac{\partial}{\partial x_{j, h}^{i}}, \\
\gamma_{j k}^{i}, \gamma_{j h k}^{i} \in C^{\infty}\left(J^{1}(F N)\right) .
\end{array}\right.
$$

Let $\Lambda^{\gamma^{2}}=L^{\gamma^{2}} \boldsymbol{v}$ be the covariant Hamiltonian density induced by a Lagrangian density $\Lambda=L \boldsymbol{v}, L \in C^{\infty}\left(J^{2}(F N)\right)$, belonging to $\mathfrak{S}_{N}$, and let $\mathcal{L}$ (resp. $\mathcal{L}^{\gamma^{2}}$ ) be the canonical Lagrangian associated with $\Lambda$ (resp. $\Lambda^{\gamma^{2}}$ ). We also assume that $\mathcal{L}$ is $\mathfrak{X}(N)$-invariant. According to (36), (37), (39), and taking (41) into account, we have

$$
L^{\gamma^{2}}=\left(\gamma_{j k}^{i}+x_{j, k}^{i}\right) L_{i}^{j k}+\left(\gamma_{j h k}^{i}+x_{j,(h k)}^{i}\right) L_{i}^{j h k}-L,
$$

$L_{i}^{j k}=\frac{\partial L}{\partial x_{j, k}^{i}}-\frac{1}{2-\delta_{h k}}\left(\frac{\partial^{2} L}{\partial x^{h} \partial x_{j,(h k)}^{i}}+x_{m, h}^{l} \frac{\partial^{2} L}{\partial x_{m}^{l} \partial x_{j,(h k)}^{i}}+x_{m,(h r)}^{l} \frac{\partial^{2} L}{\partial x_{m, r}^{l} \partial x_{j,(h k)}^{i}}\right)$,

$L_{i}^{j h k}=\frac{1}{2-\delta_{h k}} \frac{\partial L}{\partial x_{j,(h k)}^{i}}$,

where, in the present case, the Greek index $\alpha$ is a pair $(i, j)$ of Latin indices. Accordingly, if we set

$$
\begin{gathered}
\mathcal{L}_{i}^{j k}=\frac{\partial \mathcal{L}}{\partial x_{j, k}^{i}} \\
-\frac{1}{2-\delta_{h k}}\left(x_{m, h}^{l} \frac{\partial^{2} \mathcal{L}}{\partial x_{m}^{l} \partial x_{j,(h k)}^{i}}-x_{m, h}^{l} y_{l}^{m} \frac{\partial \mathcal{L}}{\partial x_{j,(h k)}^{i}}+x_{m,(h r)}^{l} \frac{\partial^{2} \mathcal{L}}{\partial x_{m, r}^{l} \partial x_{j,(h k)}^{i}}\right), \\
\mathcal{L}_{i}^{j h k}=\frac{1}{2-\delta_{h k}} \frac{\partial \mathcal{L}}{\partial x_{j,(h k)}^{i}},
\end{gathered}
$$

then the equation (42) can equivalently be written as

$$
\mathcal{L}^{\gamma^{2}}=\left(\gamma_{j k}^{i}+x_{j, k}^{i}\right) \mathcal{L}_{i}^{j k}+\left(\gamma_{j h k}^{i}+x_{j,(h k)}^{i}\right) \mathcal{L}_{i}^{j h k}-\mathcal{L}
$$

From now on, we confine ourselves to consider second-order Ehresmann connections induced from a 2-jet field, i.e., $\gamma_{\sigma}^{2}=\sigma^{*} \vartheta^{2}$, where $\sigma: J^{1}(F N) \rightarrow J^{2}(F N)$ is a section of $\pi^{21}$, and $\vartheta^{2}$ is the structure form on $J^{2}(F N)$. From the formula (14), we deduce $\gamma_{j k}^{i}=-x_{j, k}^{i}, \gamma_{j h k}^{i}=-x_{j,(h k)}^{i} \circ \sigma$, 
and hence

$$
\gamma_{\sigma}^{2}=\left(d x_{j}^{i}-x_{j, k}^{i} d x^{k}\right) \otimes \frac{\partial}{\partial x_{j}^{i}}+\left(d x_{j, h}^{i}+\gamma_{j h k}^{i} d x^{k}\right) \otimes \frac{\partial}{\partial x_{j, h}^{i}} .
$$

As $\gamma_{j h k}^{i}=\gamma_{j k h}^{i}$, from the formulas (43), (44) we finally obtain

$$
\mathcal{L}^{\gamma_{\sigma}^{2}}=\sum_{h \leq k}\left(\gamma_{j h k}^{i}+x_{j,(h k)}^{i}\right) \frac{\partial \mathcal{L}}{\partial x_{j,(h k)}^{i}}-\mathcal{L} .
$$

Proposition 7.1. The covariant Hamiltonian $\Lambda^{\gamma_{\sigma}^{2}}$ of every $\mathfrak{X}(N)$ invariant Lagrangian $\mathcal{L}$ in the class $\mathfrak{S}_{N}$, is $\mathfrak{X}(N)$-invariant if and only if all the functions $\left(\mathcal{L}_{a b, d}^{c}\right)^{\gamma_{\sigma}^{2}}, a<b, a \leq d$, are $\mathfrak{X}(N)$-invariant.

Proof. If the covariant Hamiltonian $\Lambda^{\gamma_{\sigma}^{2}}$ of every $\mathfrak{X}(N)$-invariant Lagrangian $\mathcal{L}$ is $\mathfrak{X}(N)$-invariant, then all the functions $\left(\mathcal{L}_{a b, d}^{c}\right)^{\gamma_{\sigma}^{2}}, a<b, a \leq d$, are, in particular, $\mathfrak{X}(N)$-invariant. Next, we prove the converse. If $X=$ $=u^{i} \partial / \partial x^{i}, u^{i} \in C^{\infty}(N)$, then from the formulas (11), (12) for $r=2$, we obtain

$$
\tilde{X}^{(2)}=u^{i} \frac{\partial}{\partial x^{i}}+v_{j}^{i} \frac{\partial}{\partial x_{j}^{i}}+v_{j k}^{i} \frac{\partial}{\partial x_{j, k}^{i}}+v_{j a b}^{i} \frac{\partial}{\partial x_{j,(a b)}^{i}},
$$

where $v_{j}^{i}$ and $v_{j k}^{i}$ are given in (30) and $v_{j a b}^{i} \in C^{\infty}\left(J^{2}(F N)\right)$ is given by

$$
\begin{aligned}
v_{j a b}^{i}=\frac{\partial u^{i}}{\partial x^{l}} x_{j,(a b)}^{l}-\frac{\partial u^{l}}{\partial x^{a}} x_{j,(b l)}^{i}-\frac{\partial u^{l}}{\partial x^{b}} x_{j,(a l)}^{i}-\frac{\partial^{2} u^{l}}{\partial x^{a} \partial x^{b}} x_{j, l}^{i} \\
+\frac{\partial^{2} u^{i}}{\partial x^{a} \partial x^{l}} x_{j, b}^{l}+\frac{\partial^{2} u^{i}}{\partial x^{b} \partial x^{l}} x_{j, a}^{l}+\frac{\partial^{3} u^{i}}{\partial x^{a} \partial x^{b} \partial x^{l}} x_{j}^{l} .
\end{aligned}
$$

Therefore, for every Lagrangian $\mathcal{L} \in \mathfrak{S}_{N}$, from the formulas (46), (47) we obtain, after some computations,

$$
\tilde{X}^{(2)}\left(\mathcal{L}^{\gamma_{\sigma}^{2}}\right)=\sum_{h \leq k} C_{j h k}^{i}(\tilde{X}) \frac{\partial \mathcal{L}}{\partial x_{j,(h k)}^{i}}
$$

where

$$
C_{j h k}^{i}(\tilde{X})=\tilde{X}^{(1)} \gamma_{j h k}^{i}+v_{j h k}^{i}-\left(\gamma_{s t u}^{r}+x_{s,(t u)}^{r}\right) \frac{\partial v_{j h k}^{i}}{\partial x_{s,(t u)}^{r}} .
$$

As mentioned in Section 4, every invariant Lagrangian on $J^{2}(F N)$ can be written as a smooth function of the Lagrangians $\mathcal{L}_{a b}^{c}, \mathcal{L}_{a b, d}^{c}$ defined in the formulas (18), (19). Accordingly, we can write $\mathcal{L}=\Psi \circ \Pi^{2}$, for a certain function $\Psi \in C^{\infty}\left(\mathbb{R}^{\frac{1}{6} n^{2}(n-1)(2 n+5)}\right)$, where $\Pi^{2}: J^{1}(F N) \rightarrow \mathbb{R}^{\frac{1}{6} n^{2}(n-1)(2 n+5)}$ is the 
map whose components are the functions $\mathcal{L}_{a b}^{c}, \mathcal{L}_{a b, d}^{c}$, i.e., $t_{a b}^{c} \circ \Pi^{2}=\mathcal{L}_{a b}^{c}$, $t_{a b, d}^{c} \circ \Pi^{2}=\mathcal{L}_{a b, d}^{c}$ where $\left(t_{a b}^{c}, t_{a b, d}^{c}\right), a<b, a \leq d$, are the standard coordinates on $\mathbb{R}^{\frac{1}{6}} n^{2}(n-1)(2 n+5)$. Hence, substituting

$$
\frac{\partial \mathcal{L}}{\partial x_{j,(h k)}^{i}}=\left(\frac{\partial \Psi}{\partial t_{a b, d}^{c}} \circ \Pi^{2}\right) \frac{\partial \mathcal{L}_{a b, d}^{c}}{\partial x_{j,(h k)}^{i}}
$$

into the equation (48), we obtain

$$
\tilde{X}^{(2)}\left(\mathcal{L}^{\gamma_{\sigma}^{2}}\right)=\left(\frac{\partial \Psi}{\partial t_{a b, d}^{c}} \circ \Pi^{2}\right) \tilde{X}^{(2)}\left(\left(\mathcal{L}_{a b, d}^{c}\right)^{\gamma_{\sigma}^{2}}\right),
$$

and the result follows.

We remark that $\mathcal{L}^{\gamma_{\sigma}^{2}}=-\mathcal{L}$ for every first-order Lagrangian $\mathcal{L}$ on $F N$; hence, $\tilde{X}^{(2)}\left(\mathcal{L}^{\gamma_{\sigma}^{2}}\right)=0$ if $\mathcal{L} \in C^{\infty}\left(J^{1}(F N)\right)$ is $\mathfrak{X}(N)$-invariant.

Proposition 7.2. If $\gamma_{\sigma}^{2}$ is a second-order Ehresmann connection induced from a 2-jet field $\sigma$, satisfying $L_{\tilde{X}^{(1)}} \gamma_{\sigma}^{2}=0, \forall X \in X(N)$, then the covariant Hamiltonian $\Lambda^{\nu_{\sigma}^{2}}$ of every $\mathfrak{X}(N)$-invariant Lagrangian $\mathcal{L}$ in the class $\mathfrak{C}_{N}$, is $\mathfrak{X}(N)$-invariant.

Proof. From the expression of $\gamma_{\sigma}^{2}$ in the formula (45) and the formula (30), we obtain

$$
L_{\tilde{X}^{(1)}} \gamma_{\sigma}^{2}=C_{j k l}^{i}(\tilde{X}) d x^{l} \otimes \frac{\partial}{\partial x_{j, k}^{i}},
$$

where $C_{j k l}^{i}(\tilde{X})$ is given in the formula (49). Hence, from (48) and (50) the result follows.

Unfortunately, there is no second-order Ehresmann connection $\gamma_{\sigma}^{2}$ satisfying $L_{\tilde{X}^{(1)}} \gamma_{\sigma}^{2}=0, \forall X \in X(N)$. In fact, if a point $j_{x}^{1} s \in J^{1}(F N)$ and a coordinate system $x^{i}$ centred at $x$ and adapted to $s(x)$ are given, from the formula (30) we conclude that the vector field $\tilde{X}^{(1)}$ for $u^{i}(x)=0,\left(\partial u^{i} / \partial x^{j}\right)(x)=0$, $\left(\partial^{2} u^{i} / \partial x^{j} \partial x^{k}\right)(x)=0$ for all $i, j, k$, but $\left(\partial^{3} u^{i} / \partial x^{j} \partial x^{k} \partial x^{h}\right)(x) \neq 0$ for some particular indices $i, j, k, h$, satisfies the following equation, for such indices:

$$
C_{j k h}^{i}(\tilde{X})\left(j_{x}^{1} s\right)=\frac{\partial^{3} u^{i}}{\partial x^{j} \partial x^{k} \partial x^{h}}(x) \neq 0 .
$$

From the formula (50), we deduce that $L_{\tilde{X}^{(1)}} \gamma_{\sigma}^{2}$ is a section of the vector bundle $\left(\pi^{1}\right)^{*} T^{*} N \otimes V\left(\pi^{10}\right)$. As $\pi^{10}: J^{1}(F N) \rightarrow F N$ is an affine bundle modelled over $\pi^{*} T^{*} N \otimes V(\pi)$ we have a natural isomorphism of vector bundles, 
$\left(\pi^{10}\right)^{*}\left(\pi^{*} T^{*} N \otimes V(\pi)\right) \rightarrow V\left(\pi^{10}\right)$. Moreover, from the isomorphism defined in (35), we deduce an isomorphism $\left(\pi^{10}\right)^{*} V(\pi) \rightarrow\left(\pi^{1}\right)^{*}\left(T^{*} N \otimes T N\right)$, which allows us to consider $L_{\tilde{X}^{(1)}} \gamma_{\sigma}^{2}$ as being the section of $\left(\pi^{1}\right)^{*}\left(\otimes^{3} T^{*} N \otimes T N\right)$, given by $L_{\tilde{X}^{(1)}} \gamma_{\sigma}^{2}=C_{j k l}^{i}(\tilde{X}) y_{s}^{j} d x^{l} \otimes d x^{k} \otimes d x^{s} \otimes \partial / \partial x^{i}$, and we obtain

Proposition 7.3. The covariant Hamiltonian $\Lambda^{\gamma_{\sigma}^{2}}$ of every $\mathfrak{X}(N)$ invariant Lagrangian $\mathcal{L} \in \mathfrak{C}_{N}$ is $\mathfrak{X}(N)$-invariant if and only if, $\operatorname{alt}_{23}\left(L_{\tilde{X}^{(1)}} \gamma_{\sigma}^{2}\right)=0, \forall X \in \mathfrak{X}(N)$, where alt $_{i j}$ denotes the antisymmetrization operator in the $i$-th and $j$-th covariant arguments.

PRoof. By setting $X_{c}=x_{c}^{i} \partial / \partial x^{i}$, the previous formula for $L_{\tilde{X}^{(1)}} \gamma_{\sigma}^{2}$ can be rewritten as follows: $L_{\tilde{X}^{(1)}} \gamma_{\sigma}^{2}=x_{d}^{l} x_{a}^{k} C_{b k l}^{i}(\tilde{X}) y_{i}^{c} \theta^{d} \otimes \theta^{a} \otimes \theta^{b} \otimes X_{c}$. Moreover, as it is easily checked, we have $\operatorname{alt}_{12}\left(L_{\tilde{X}^{(1)}} \gamma_{\sigma}^{2}\right)=0$, and

$$
\operatorname{alt}_{23}\left(L_{\tilde{X}^{(1)}} \gamma_{\sigma}^{2}\right)=x_{d}^{l} y_{i}^{c}\left(x_{a}^{k} C_{b k l}^{i}(\tilde{X})-x_{b}^{k} C_{a k l}^{i}(\tilde{X})\right) \theta^{d} \otimes \theta^{a} \wedge \theta^{b} \otimes X_{c} .
$$

Taking the expression of the Lagrangians $\mathcal{L}_{a b, d}^{c}$ given in (19) and the formula

$$
\frac{\partial \mathcal{L}_{a b, d}^{c}}{\partial x_{j,(h k)}^{i}}=x_{d}^{r}\left(x_{a}^{v} \delta_{b}^{j}-x_{b}^{v} \delta_{a}^{j}\right) \delta_{(r v)}^{(h k)} y_{i}^{c}
$$

into account, from the formula (48) we have

$$
\begin{aligned}
\tilde{X}^{(2)}\left(\mathcal{L}_{a b, d}^{c}\right)^{\gamma_{\sigma}^{2}} & =\sum_{h \leq k} C_{j k h}^{i}(\tilde{X}) \frac{\partial \mathcal{L}_{a b, d}^{c}}{\partial x_{j,(h k)}^{i}} \\
& =x_{d}^{l}\left(x_{a}^{k} C_{b k l}^{i}(\tilde{X})-x_{b}^{k} C_{a k l}^{i}(\tilde{X})\right) y_{i}^{c} .
\end{aligned}
$$

Hence, the equation (51) can be written as follows:

$$
\operatorname{alt}_{23}\left(L_{\tilde{X}^{(1)}} \gamma_{\sigma}^{2}\right)=\tilde{X}^{(2)}\left(\mathcal{L}_{a b, d}^{c}\right)^{\gamma_{\sigma}^{2}} \theta^{d} \otimes \theta^{a} \wedge \theta^{b} \otimes X_{c} .
$$

This equation and Lemma 7.1 allow us to conclude the proof.

Theorem 7.4. Let $\tau_{N}^{1}, \tau_{N}^{2}$ be the mappings given by,

$$
\begin{gathered}
\tau_{N}^{1}: J^{1}(F N) \rightarrow \wedge^{2} T^{*} N \otimes T N, \\
\tau_{N}^{1}\left(j_{x}^{1} s\right)=\left(\operatorname{Tor}_{\nabla^{s}}\right)_{x}, \\
\tau_{N}^{2}: J^{2}(F N) \rightarrow T^{*} N \otimes \wedge^{2} T^{*} N \otimes T N, \\
\tau_{N}^{2}\left(j_{x}^{2} s\right)=\left(\nabla^{s} \operatorname{Tor}_{\nabla^{s}}\right)_{x},
\end{gathered}
$$

where $\nabla^{s}$ is the linear connection parallelizing the linearframe defined by the section s. Let $\gamma_{\sigma}^{2}=\sigma^{*} \vartheta^{2}$ be the second-order Ehresmann connection induced 
by a 2-jet field $\sigma: J^{1}(F N) \rightarrow J^{2}(F N)$. The covariant Hamiltonian $\Lambda^{\gamma_{\sigma}^{2}}$ associated with every $\mathfrak{X}(N)$-invariant Lagrangian density $\Lambda$ on $J^{2}(F N)$ in the class $\mathfrak{S}_{N}$ is $\mathfrak{X}(N)$-invariant, if and only if $\gamma_{\sigma}^{2}$ satisfies the following equation:

$$
\operatorname{alt}_{23}\left(\operatorname{hor} \gamma_{\sigma}^{2}\right)=-\tau_{N}^{2}+\Phi \circ\left(\pi^{20}, \tau_{N}^{1} \circ \pi^{21}\right),
$$

where $\Phi: \pi^{*}\left(\wedge^{2} T^{*} N \otimes T N\right) \rightarrow \pi^{*}\left(T^{*} N \otimes \wedge^{2} T^{*} N \otimes T N\right)$ is the map induced by a system of functions $\Phi_{a b d}^{c} \in C^{\infty}\left(\wedge^{2}\left(\mathbb{R}^{n}\right)^{*} \otimes \mathbb{R}^{n}\right), a<b$; i.e.,

$$
\Phi(u, \tau)=\left(u, \sum_{a<b} \Phi_{a b d}^{c}\left(\left(\wedge^{2} u^{*} \otimes u^{-1}\right)(\tau)\right) \omega^{d} \otimes \omega^{a} \wedge \omega^{b} \otimes X_{c}\right)
$$

and here $u=\left(X_{1}, \ldots, X_{n}\right) \in F_{x} N$ is considered to be a linear isomorphism $u: \mathbb{R}^{n} \rightarrow T_{x} N$, with dual coframe $\left(\omega^{1}, \ldots, \omega^{n}\right)$, and $\tau \in \wedge^{2} T_{x}^{*} N \otimes T_{x} N$. For every manifold $N$, 2-jet fields $\sigma: J^{1}(F N) \rightarrow J^{2}(F N)$ globally defined on $J^{1}(F N)$ whose induced second-order Ehresmann connection $\gamma_{\sigma}^{2}$ satisfies the equation (53), always exist.

PRoOF. Letting $\bar{\Delta}_{a b d}^{c}=x_{d}^{l} y_{i}^{c}\left(x_{a}^{k} \gamma_{b k l}^{i}-x_{b}^{k} \gamma_{a k l}^{i}\right) \in C^{\infty}\left(J^{1}(F N)\right)$, from the formula (52) we obtain

$$
\sum_{h \leq k} \gamma_{j h k}^{i} \frac{\partial \mathcal{L}_{a b, d}^{c}}{\partial x_{j,(h k)}^{i}}=\bar{\Delta}_{a b d}^{c}
$$

From the formula (46), we then deduce

$$
\left(\mathcal{L}_{a b, d}^{c}\right)^{\gamma_{\sigma}^{2}}=\overline{\lrcorner}_{a b d}^{c}+\sum_{h \leq k} x_{j,(h k)}^{i} \frac{\partial \mathcal{L}_{a b, d}^{c}}{\partial x_{j,(h k)}^{i}}-\mathcal{L}_{a b, d}^{c} \in C^{\infty}\left(J^{1}(F N)\right) .
$$

Hence, $\tilde{X}^{(2)}\left(\mathcal{L}_{a b, d}^{c}\right)^{\gamma_{\sigma}^{2}}=\tilde{X}^{(1)}\left(\mathcal{L}_{a b, d}^{c}\right)^{\gamma_{\sigma}^{2}}$, and $\tilde{X}^{(1)}\left(\mathcal{L}_{a b, d}^{c}\right)^{\gamma_{\sigma}^{2}}=0$ for every vector field $X \in X(N)$ if and only if,

$$
\bar{\Delta}_{a b d}^{c}+\sum_{h \leq k} x_{j,(h k)}^{i} \frac{\partial \mathcal{L}_{a b, d}^{c}}{\partial x_{j,(h k)}^{i}}-\mathcal{L}_{a b, d}^{c}=\Phi_{a b d}^{c} \circ \Pi^{1},
$$

where $\Pi^{1}: J^{1}(F N) \rightarrow \mathbb{R}^{\frac{1}{2} n^{2}(n-1)}$ is the map introduced in the proof of Lemma 5.3. By setting $\Delta_{s k l}^{i}=y_{s}^{j} \gamma_{j k l}^{i}-y_{k}^{j} \gamma_{j l l}^{i}$, we obtain

$$
x_{a}^{k} x_{b}^{s} x_{d}^{l} y_{i}^{c} \Delta_{s k l}^{i}=\bar{\Delta}_{a b d}^{c} .
$$

Hence, by multiplying the equation (55) by $x_{c}^{i^{\prime}} y_{k^{\prime}}^{a} y_{s^{\prime}}^{b} y_{l^{\prime}}^{d}$, and taking the formula (56) and

$$
\sum_{h \leq k} x_{j,(h k)}^{i} \frac{\partial \mathcal{L}_{a b, d}^{c}}{\partial x_{j,(h k)}^{i}}=x_{d}^{l} y_{i}^{c}\left(x_{a}^{k} x_{b,(k l)}^{i}-x_{b}^{k} x_{a,(k l)}^{i}\right)
$$


into account, we obtain

$$
\begin{aligned}
\Delta_{s^{\prime} k^{\prime} l^{\prime}}^{i^{\prime}} & =x_{c}^{i^{\prime}} y_{k^{\prime}}^{a} y_{s^{\prime}}^{b} y_{l^{\prime}}^{d}\left(\mathcal{L}_{a b, d}^{c}-\sum_{h \leq k} x_{j,(h k)}^{i} \frac{\partial \mathcal{L}_{a b, d}^{c}}{\partial x_{j,(h k)}^{i}}+\Phi_{a b d}^{c} \circ \Pi^{1}\right) \\
& =x_{c}^{i^{\prime}} y_{k^{\prime}}^{a} y_{s^{\prime}}^{b} y_{l^{\prime}}^{d}\left(\mathcal{L}_{a b, d}^{c}+\Phi_{a b d}^{c} \circ \Pi^{1}\right)-\left(y_{s^{\prime}}^{b} x_{b,\left(k^{\prime} l^{\prime}\right)}^{i^{\prime}}-y_{k^{\prime}}^{a} x_{a,\left(s^{\prime} l^{\prime}\right)}^{i^{\prime}}\right) .
\end{aligned}
$$

As $\pi^{10}: J^{1}(F N) \rightarrow F N$ is an affine bundle modelled over $\pi^{*} T^{*} N \otimes V(\pi)$ there is an isomorphism of vector bundles, $\left(\pi^{10}\right)^{*}\left(\pi^{*} T^{*} N \otimes V(\pi)\right) \rightarrow V\left(\pi^{10}\right)$, given by $d x^{k} \otimes \partial / \partial x_{j}^{i} \mapsto \partial / \partial x_{j, k}^{i}$. Moreover, from the isomorphism defined in (35), we deduce an isomorphism $\left(\pi^{10}\right)^{*} V(\pi) \rightarrow\left(\pi^{1}\right)^{*}\left(T^{*} N \otimes T N\right)$. Hence, by composing both isomorphisms, we obtain an isomorphism

$$
\begin{aligned}
V\left(\pi^{10}\right) & \rightarrow\left(\pi^{1}\right)^{*}\left(T^{*} N \otimes T^{*} N \otimes T N\right), \\
\frac{\partial}{\partial x_{j, k}^{i}} & \mapsto y_{l}^{j} d x^{k} \otimes d x^{l} \otimes \frac{\partial}{\partial x^{i}} .
\end{aligned}
$$

Hence, the horizontalization of $\gamma_{\sigma}^{2}$ in the formula (45), yields

$$
\operatorname{hor}\left(\gamma_{\sigma}^{2}\right)=y_{l}^{j}\left(x_{j,(h k)}^{i}+\gamma_{j h k}^{i}\right) d x^{k} \otimes d x^{h} \otimes d x^{l} \otimes \frac{\partial}{\partial x^{i}},
$$

and by taking the antisymmetric part of hor $\gamma_{\sigma}^{2}$ on its 2 nd and $3 r d$ covariant arguments, we obtain a fibred morphism over $N$,

$$
\operatorname{alt}_{23}\left(\operatorname{hor} \gamma_{\sigma}^{2}\right): J^{2}(F N) \rightarrow \wedge^{2} T^{*} N \otimes T N,
$$

locally given by

$$
\begin{aligned}
\operatorname{alt}_{23}\left(\text { hor } \gamma_{\sigma}^{2}\right) & =y_{l}^{j}\left(x_{j,(h k)}^{i}+\gamma_{j h k}^{i}\right) d x^{k} \otimes d x^{h} \wedge d x^{l} \otimes \frac{\partial}{\partial x^{i}} \\
& =\sum_{h<l} A_{k h l}^{i} d x^{k} \otimes d x^{h} \wedge d x^{l} \otimes \frac{\partial}{\partial x^{i}},
\end{aligned}
$$

where $A_{k h l}^{i}=y_{l}^{j} x_{j,(h k)}^{i}-y_{h}^{j} x_{j,(k l)}^{i}+\Delta_{l h k}^{i}$. From the equation (57) we deduce

$$
A_{l^{\prime} k^{\prime} s^{\prime}}^{i^{\prime}}=x_{c}^{i^{\prime}} y_{k^{\prime}}^{a} y_{s^{\prime}}^{b} y_{l^{\prime}}^{d} \mathcal{L}_{a b, d}^{c}+x_{c}^{i^{\prime}} y_{k^{\prime}}^{a} y_{s^{\prime}}^{b} y_{l^{\prime}}^{d}\left(\Phi_{a b d}^{c} \circ \Pi^{1}\right),
$$

and from the formula (20) we obtain

$$
\begin{aligned}
\tau_{N}^{2}\left(j_{x}^{2} s\right) & =-\mathcal{L}_{a b, d}^{c}\left(j_{x}^{2} s\right)\left(\omega^{d} \otimes \omega^{a} \wedge \omega^{b} \otimes X_{c}\right)_{x} \\
& =-\left(y_{l^{\prime}}^{d} y_{k^{\prime}}^{a} y_{s^{\prime}}^{b} x_{c}^{i^{\prime}} \mathcal{L}_{a b, d}^{c} d x^{l^{\prime}} \otimes d x^{k^{\prime}} \wedge d x^{s^{\prime}} \otimes \frac{\partial}{\partial x^{i^{\prime}}}\right)\left(j_{x}^{2} s\right) .
\end{aligned}
$$

Hence, from the equations (58), (59), and the definition of the map $\Phi$ given in (54), the equations (53) follow. 
Finally, for $\Phi_{a b d}^{c}=0$, for all the indices $a<b, c, d$, the equation (53) becomes alt ${ }_{23}\left(\right.$ hor $\left.\gamma_{\sigma}^{2}\right)=-\tau_{N}^{2}$, and the space of 2 -jet fields $\sigma$ whose induced second-order Ehresmann connection $\gamma_{\sigma}^{2}$ satisfies such equation is readily seen to be the sections of an affine bundle modelled over the vector subbundle $\left(\pi^{10}\right)^{*}\left(S^{3} T^{*} N \otimes T N\right) \subset\left(\pi^{10}\right)^{*}\left(S^{2} T^{*} N \otimes T^{*} N \otimes T N\right)$, which is naturally isomorphic to $\left(\pi^{1}\right)^{*}\left(\pi^{*} S^{2} T^{*} N \otimes V(\pi)\right)$. This concludes the proof.

\section{REFERENCES}

[1] M. F. AтіYAн, Complex analytic connections in fibre bundles, Trans. Amer. Math. Soc., 85 (1957), pp. 181-207.

[2] D. BleEcker, Gauge Theory and Variational Principles, Addison-Wesley Publishing Company, Inc., Reading, MA, 1981.

[3] M. Castrillón López - J. Muñoz Masqué, The geometry of the bundle of connections, Math. Z., 236 (2001), pp. 797-811.

[4] M. CRAMPin - E. MartíneZ - W. SARLET, Linear connections for systems of second-order ordinary differential equations, Ann. Inst. H. Poincaré Phys. Théor., 65, no. 2 (1996), pp. 223-249.

[5] D. J. EcK, Gauge-natural bundles and generalized gauge theories, Mem. Amer. Math. Soc., 247 (1981).

[6] Antonio Fernández - Pedro L. García - J. Muñoz Masqué, Gaugeinvariant covariant Hamiltonians, J. Math. Phys., 41 (2000), pp. 5292-5303.

[7] Pedro L. García, Gauge algebras, curvature and symplectic structure, J. Differential Geom., 12 (1977), pp. 209-227.

[8] Pedro L. García - J. Muñoz Masqué, Differential invariants on the bundles of linear frames, J. Geom. Phys., 7, (1990), pp. 395-418.

[9] H. Goldschmidt - S. Sternberg, The Hamilton-Cartan formalism in the calculus of variations, Ann. Inst. Fourier (Grenoble), 23, no. 1 (1973), pp. 203267.

[10] V. Guillemin - S. Sternberg, Symplectic techniques in physics, Cambridge University Press, Cambridge, UK, 1983.

[11] S. Kobayashi - K. Nomizu, Foundations of Differential Geometry, Volume I, John Wiley \& Sons, Inc., N.Y., 1963.

[12] J. L. Koszul, Fibre bundles and Differential Geometry, Tata Institute of Fundamental Research, Bombay, 1960.

[13] L. Mangiarotti - G. SARDANAShVily, Connections in classical and quantum field theory, World Scientific Publishing Co., Inc., River Edge, NJ, 2000.

[14] J. Marsden - S. ShKoller, Multisymplectic geometry, covariant Hamiltonians, and water waves, Math. Proc. Cambridge Phil. Soc., 125 (1999), pp. 553-575.

[15] E. MASSA - E. PAGANI, Jet bundle geometry, dynamical connections, and the inverse problem of Lagrangian mechanics, Ann. Inst. H. Poincaré Phys. Théor., 61, no. 1 (1994), pp. 17-62.

[16] J. MuÑoz MASQuÉ, Formes de structure et transformations infinitésimales de contact d'ordre supérieur, C.R. Acad. Sci. Paris, 298, Série I (1984), pp. 185-188. 
[17] J. Muñoz Masqué, An axiomatic characterization of the Poincaré-Cartan form for second-order variational problems, Lecture Notes in Math. 1139, Springer-Verlag, 1985, pp. 74-84.

[18] J. MuÑoz MasquÉ - M. E. Rosado, Invariant variational problems on linear frame bundles, J. Phys. A: Math. Gen., 35 (2002), pp. 2013-2036.

[19] P. J. Olver, Equivalence, Invariants, and Symmetry, Cambridge University Press, Cambridge, UK, 1995.

[20] G. A. Sardanashvily, Gauge Theory in Jet Manifolds, Hadronic Press Monographs in Applied Mathematics, Hadronic Press, Inc., Palm Harbor, FL, U.S.A., 1993.

[21] D. J. SAunders, The Geometry of Jet Bundles, Cambridge University Press, Cambridge, UK, 1989.

[22] D. J. SAunders - M. CRAmpin, On the Legendre map in higher-order field theories, J. Phys. A: Math. Gen., 23 (1990), pp. 3169-3182.

[23] R. UtiYama, Invariant theoretical interpretation of interaction, Phys. Rev., 101 (1956), 1597-1607.

Manoscritto pervenuto in redazione il 22 giugno 2006. 